\title{
Modelagem estocástica de uma população de neurônios.
}

\author{
Karina Yuriko Yaginuma
}

TESE APRESENTADA

$\mathrm{AO}$

Instituto de Matemática e Estatística

DA

Universidade DE SÃo PAUlo

PARA

OBTENÇÃO DO TÍTULO

$\mathrm{DE}$

DOUTOR EM CiÊNCIAS

Programa: Estatística

Orientador: Antonio Galves

Durante o desenvolvimento deste trabalho o autor recebeu os auxílios financeiros da CAPES e CNPq.

Esta tese foi produzida no Núcleo de Apoio à Pesquisa em Modelagem Estocástica e Complexidade/MaCLinC como parte das atividades do Centro de Pesquisa, Inovação e Difusão em Neuromatemática, financiado pela FAPESP (processo 2013/ 07699-0) e do Programa

CAPES-NUFFIC (processo 038/12).

São Paulo, maio de 2014 


\section{Modelagem estocástica de uma população de neurônios}

Esta versão da tese contém as correções e alterações sugeridas pela Comissão Julgadora durante a defesa da versão original do trabalho, realizada em 08/05/2014. Uma cópia da versão original está disponível no

Instituto de Matemática e Estatística da Universidade de São Paulo.

Comissão Julgadora:

- Prof. Dr. J. Antonio Galves (orientador) - IME-USP

- Prof. Dr. Miguel Natalio Abadi - IME-USP

- Prof. Dr. Jesús Enrique García - UNICAMP

- Prof ${ }^{\mathrm{a}}$. Dr ${ }^{\mathrm{a}}$. Nancy Lopes Garcia - UNICAMP

- Prof. Dr. Cristian Favio Coletti - UFABC 


\section{Agradecimentos}

Agradeço primeiramente aos meus pais, Marlene Hatsuyo Yaginuma e Yuji Yaginuma, pelo apoio e incentivo que sempre me deram durante todos esses anos.

Ao professor Antonio Galves, pela orientação, dedicação, apoio e por sempre estar presente durante a elaboração desta tese.

Ao professor Jesus Enrique Garcia, por ter me incentivado a entrar no mestrado e consequentemente no doutorado.

As minhas queridas irmãs, Vanessa, Erika e Simony, e aos meus queridos amigos, Letícia, Fernanda e Atahualpa, por sempre me apoirem, nos momentos bons e ruins.

Aos amigos que fiz dentro do IME-USP. Em especial, Douglas, Rodrigo, Aline e Guilherme.

Aos participantes da banca examinadora, pelas sugestões e correções.

A nossa querida secretária do NUMEC Lourdes Netto, por todo auxílio que nos proporciona todos os dias. 


\section{Resumo}

Yaginuma, K. Y. Modelagem estocástica de uma população de neurônios. Tese (Doutorado)

- Instituto de Matemática e Estatística, Universidade de São Paulo, São Paulo, 2014.

Nesta tese consideramos uma nova classe de sistemas markovianos de partículas com infinitas componentes interagentes. O sistema representa a evolução temporal dos potenciais de membrana de um conjunto infinito de neurônios interagentes. Provamos a existência e unicidade do processo construindo um pseudo-algoritmo de simulação perfeita e mostrando que este algoritmo roda em um número finito de passos quase certamente. Estudamos também o comportamento do sistema quando consideramos apenas um conjunto finito de neurônios. Neste caso, construímos um procedimento de simulação perfeita para o acoplamento entre o processo limitado a um conjunto finito de neurônios e o processo que considera todos os neurônios do sistema. Como consequência encontramos um limitante superior para a probabilidade de discrepância entre os processos.

Palavras-chave: Simulação perfeita, sistemas markovianos de partículas, redes neurais biológicas. 


\section{Abstract}

Yaginuma, K. Y. Stochastic modelling of a population of neurons. Tese (Doutorado) - Instituto de Matemática e Estatística, Universidade de São Paulo, São Paulo, 2014.

We consider a new class of interacting particle systems with a countable number of interacting components. The system represents the time evolution of the membrane potentials of an infinite set of interacting neurons. We prove the existence and uniqueness of the process, by the construction of a perfect simulation procedure. We show that this algorithm is successful, that is, we show that the number of steps of the algorithm is finite almost surely. We also study the behaviour of the system when we consider only a finite number of neurons. In this case, we construct a perfect simulation procedure for the coupling of the process with a finite number of neurons and the process with a infinite number of neurons. As a consequence we obtain an upper bound for the error we make when sampling from a finite set of neurons instead of the infinite set of neurons.

Keywords: Perfect simulation, markovian particle systems, neural nets. 


\section{Sumário}

1 Introdução $\quad 1$

1.1 O problema da modelagem estocástica de um sistema de neurônios . . . . . . . . . . 1

1.2 Principais contribuições científicas desta tese . . . . . . . . . . . . . . . 2

1.3 Organização do trabalho . . . . . . . . . . . . . . . . . . . . 2

2 Preliminares $\quad 3$

2.1 Sistemas com infinitas cadeias com memória de alcance variável interagentes . . . . . 4

2.2 Teorema de Existência e Unicidade . . . . . . . . . . . . . . . . . . . . 5

2.3 Correlação entre intervalos sucessivos entre disparos $\ldots \ldots \ldots$. . . . . . . . . 7

3 Modelo básico e o Teorema de Existência e Unicidade 9

3.1 Definições . . . . . . . . . . . . . . . . . . . . . . . . 9

3.2 Teorema de Existência e Unicidade . . . . . . . . . . . . . . . . . . . . . . . . . . . . . . . . . . . . . . . .

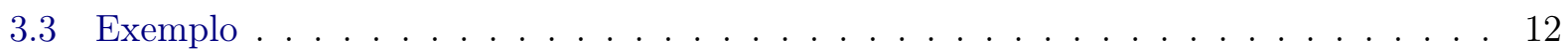

4 Uma algoritmo de simulação perfeita para o processo $\left(\xi_{t}\right)_{t \in \mathbb{R}} \mathbf{1 5}$

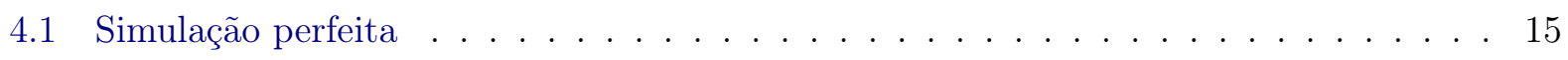

4.2 Processo rascunho para trás $\ldots \ldots \ldots \ldots \ldots \ldots \ldots$

4.3 Algoritmo de simulação perfeita . . . . . . . . . . . . . . . . . . . . 22

5 Um algoritmo de simulação perfeita para o processo acoplado $\left(\xi_{t}, \xi_{t}^{[F]}\right)_{t \in \mathbb{R}} \quad \mathbf{2 7}$

5.1 Definições e resultado . . . . . . . . . . . . . . . . . . . 27

5.2 Simulação perfeita para o aclopamento dos processos $\left(\xi_{t}, \xi_{t}^{[F]}\right)_{t \in \mathbb{R}} \ldots \ldots \ldots \ldots$

5.2 .1 Algoritmo . . . . . . . . . . . . . . . . . . . 31

6 Prova dos resultados 33

6.1 Prova do Teorema $4.3 .1 \ldots \ldots \ldots \ldots \ldots$

6.1 .1 Prova da primeira parte . . . . . . . . . . . . 33

6.1 .2 Prova da segunda parte . . . . . . . . . . . . . . . . . . 34

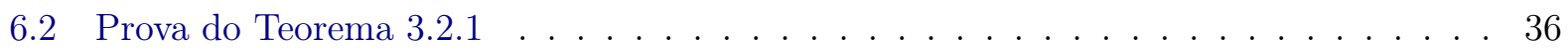

6.3 Prova do Teorema $5.1 .1 \ldots \ldots \ldots \ldots \ldots$

7 Conclusão $\quad 39$

$\begin{array}{ll}\text { Referências Bibliográficas } & 41\end{array}$ 


\section{Capítulo 1}

\section{Introdução}

\subsection{O problema da modelagem estocástica de um sistema de neurô- nios}

A atividade de um neurônio é manisfestada pela emissão de potenciais de ação ou disparos. Tal atividade é realizada por cada neurônio dentro de um sistema composto por um número grande de neurônios interagentes. Uma estimativa do número de neurônios no cérebro humano é de cerca de 100 bilhões. Além disso, é estimado que um único neurônio pode fazer cerca de 10 mil conexões distintas com outros neurônios do sistema.

O neurônio é constituído pelas seguintes partes: corpo celular, o núcleo celular, dendritos (prolongamentos numerosos e curtos do corpo celular, receptores de mensagens) e o axônio (prolongamento que transmite o impulso nervoso proveniente do corpo celular).

Enquanto a forma do potencial é essencialmente constante para um dado neurônio, de tal maneira que é possível distinguir cada neurônio pelo formato do seu potencial de ação, a sucessão de disparos que o neurônio é capaz de emitir depende do seu estado, dos estímulos proveniente de outros neurônios, como também de estímulos externos ao sistema.

O potencial de ação ocorre com uma probabilidade que é uma função crescente da concentração de íons positivos dentro da célula, após a emissão do potencial de ação o neurônio perde carga positiva e volta a um estado de repouso. A diferença da concentração de íons entre o interior e exterior da célula é chamada de potencial de membrana.

Um processo pelo qual um neurônio pode estimular ou inibir a ocorrência de um disparo de outro neurônio pode ser descrito da seguinte maneira. Com o disparo de um neurônio, neurotransmissores são emitidos a partir do seu axônio terminal. Se outro neurônio está próximo, os neurotransmissores podem agir em seus dentritos alterando a permeabilidade do neurônio e proporcionando a entrada de íons na célula, essa troca de íons pode estimular ou inibir o outro neurônio.

Essa é a motivação para a introdução de uma classe de sistemas de partículas com interações de alcance infinito que consideramos nessa tese. Para lidar com o problema do grande número de neurônios parece natural considerar um sistema com um conjunto enumerável de componentes com interações de alcance infinito.

O sistema descreve a evolução dos potenciais de membrana dos neurônios, em tempo contínuo. Essa classe de sistemas é uma extensão da classe de modelos introduzida em Galves e Löcherbach (2013). Eles consideram um sistema cuja probabilidade de disparo de um dado neurônio depende da atividade acumulada do sistema depois do instante do seu último disparo. A evolução temporal do sistema é em tempo discreto, cada neurônio é caracterizado pela ocorrência ou não de um disparo, com o símbolo 1 se houve um disparo e 0 se não, em cada instante. Se olharmos para um único neurônio, a evolução temporal parece uma cadeia estocástica com memória de alcance variável, em que o neurônio precisa olhar para o passado até encontrar o seu último disparo.

Cessac (2011) sugeriu o mesmo tipo de dependência do passado. Esse artigo considera um sistema com um número finito de neurônios, em que cada neurônio é caracterizado pela evolução do seu potencial de membrana. A imagem desse processo no qual apenas os tempos de disparos 
são registrados também é uma cadeia estocástica com memória de alcance variável, cujo alcance depende do último disparo de cada neurônio. Em nosso caso, vamos considerar um sistema com um número infinito de neurônios caracterizados pelos seus potenciais de membrana.

Sistemas finitos de processos pontuais em tempo discreto ou contínuo usados para descrever sistemas biológicos neurais possui uma longa história cujo ponto de partida foi provavelmente Hawkes (1971) do ponto de vista probabilístico e Brillinger (1988) do ponto de vista estatístico.

\subsection{Principais contribuições científicas desta tese}

As principais contribuições deste trabalho são:

- Introdução de uma nova classe de modelos, esta é uma extensão da classe de processos introduzido por Galves e Löcherbach (2013), capaz de descrever a evolução de um sistema com infinitas componentes e suas interações.

- Desenvolvimento de um procedimento de simulação perfeita para a nova classe de modelos introduzida nesta tese.

- Desenvolvimento de um procedimento de simulação perfeita para o acoplamento entre o processo com infinitas componentes e o processo restrito a um conjunto com um número finito de componentes.

- Obtenção de um limitante superior para a probabilidade da discrepância entre os dois processos, para um ponto e um instante dados, quando utilizamos o algoritmo de simulação perfeita para gerar uma amostra do processo acoplado.

\subsection{Organização do trabalho}

Esta tese está organizada da seguinte maneira. No Capítulo 2 introduzimos o modelo definido em Galves e Löcherbach (2013) e enunciamos alguns resultados sobre o modelo. No Capítulo 3 apresentamos a formulação matemática do modelo e as condições sob as quais temos a existência e unicidade do mesmo.

A principal ferramenta utilizada para provar a existência e unicidade do modelo é o procedimento de simulação perfeita apresentado no Capítulo 4. No Capítulo 5, construímos um procedimento de simulação perfeita para acoplar dois processos, o processo com um conjunto infinito de neurônios com o processo truncado no qual só se considera um conjunto finito de neurônios. A prova de todos os resultados são apresentados no Capítulo 6. 


\section{Capítulo 2}

\section{Preliminares}

O modelo que propomos nesta tese é baseado no modelo introduzido por Galves e Löcherbach (2013). Eles consideram uma nova classe de processos não markovianos com um conjunto enumerável de neurônios. A principal motivação do modelo introduzido em Galves e Löcherbach (2013) é a modelagem estocástica de sistemas neurais biológicos, onde cada componente representa a atividade de um neurônio. Estes sistemas evoluem no tempo, e a evolução temporal não é descrita por um processo de Markov.

Precisamente, quando um neurônio emite um potencial de ação ele perde carga e portanto volta para um estado de repouso, reiniciando o acúmulo de carga positiva para eventualmente emitir um novo potencial de ação. Isto implica que o processo é não markoviano, mas sim um processo com memório de alcance variável.

O modelo considera que cada neurônio do sistema depende de uma porção variável do passado. Isto é, a probabilidade de disparo de cada neurônio depende da atividade acumulada dos neurônios que o afetam depois do instante do seu último disparo. Eles consideram também que a probabilidade de disparo também depende do tempo decorrido desde o último disparo do neurônio.

A evolução do sistema é em tempo discreto. Para cada instante $t \in \mathbb{Z}$, a probabilidade de ocorrer um disparo no instante $t$ em cada neurônio do sistema é independente dos outros neurônios, dado todo o passado do sistema. Quando observamos a evolução temporal de um único neurônio, podemos dizer que a evolução parece com uma cadeia estocástica com memória de alcance variável.

No artigo eles mostraram que o modelo tem um comportamento compatível com resultados empíricos presentes na literatura da neurociência. Em Gerstner e Kistler (2002), citando Goldberg et al. (1955), eles observaram que, em muitos dados experimentais, a correlação empírica entre intervalos sucessivos entre disparos é muito pequena, indicando que a descrição de uma sequência de disparos como um processo estacionário de renovação é uma boa aproximação. No entanto, no artigo Nawrot et al. (2007) encontraram-se evidências estatistícas de que intervalos sucessivos entre disparos são negativamente correlacionados. Em Galves e Löcherbach (2013), eles mostraram que estes dois fatos contraditórios podem ser explicados pelo modelo.

Neste capítulo, vamos definir o modelo introduzido por Galves e Löcherbach (2013). Apresentaremos também dois teoremas que provam a existência e unicidade dos sistemas com infinitas cadeias com memória de alcance variável interagentes, sob diferentes condições. Finalizamos o capítulo enunciando o resultado sobre a correlação entre intervalos sucessivos entre disparos. 


\subsection{Sistemas com infinitas cadeias com memória de alcance variável interagentes}

Seja $\left(X_{t}\right)_{t \in \mathbb{Z}}$ uma cadeia estocástica assumindo valores em $\{0,1\}^{\mathcal{I}}$ para algum conjunto enumerável de neurônios $\mathcal{I}$, definida em um espaço de probabilidade $(\Omega, \mathcal{A}, P)$ adequado. Para cada neurônio $i$ em cada instante $t \in \mathbb{Z}$,

$$
X_{t}(i)= \begin{cases}1, & \text { se ocorreu um disparo do neurônio } i \text { no instante } t \\ 0, & \text { caso contrário. }\end{cases}
$$

A configuração global dos neurônios no instante $t$ é denotado por $X_{t}=\left(X_{t}(i), i \in \mathcal{I}\right)$. Defina a seguinte filtração

$$
\mathcal{F}_{t}=\sigma\left(X_{s}, s \leq t\right), \text { para } t \in \mathbb{Z} .
$$

Para cada neurônio $i \in \mathcal{I}$ e cada instante $t \in \mathbb{Z}$, seja

$$
L_{t}^{(i)}=\sup \left\{s<t: X_{s}(i)=1\right\}
$$

o instante do último disparo do neurônio $i$.

As interações entre os neurônios são definidas a partir de uma família de pesos sinápticos

$$
\left\{W_{j \rightarrow i}, \text { para } i, j \in \mathcal{I}\right\},
$$

onde $W_{j \rightarrow i} \in \mathbb{R}$ para todo par $j \rightarrow i$. Vamos supor que $W_{j \rightarrow j}=0$ para todo $j \in \mathcal{I}$.

Dizemos que $W_{j \rightarrow i}$ é o peso sináptico do neurônio $j$ em $i$. Se $W_{j \rightarrow i}>0$, então o neurônio $j$ possui um efeito excitatório sobre o neurônio $i$. Já no caso em que $W_{j \rightarrow i}<0, j$ possui um efeito inibitório sobre $i$.

Agora podemos definir a dinâmica do processo. Em cada instante $t \in \mathbb{Z}$, condicionado a todo o passado, a probabilidade de um disparo em cada neurônio independe do que acontece com os outros neurônios do sistema no instante $t$. Isto significa que para qualquer subconjunto finito $F \in \mathcal{I}$ e $a_{i} \in\{0,1\}$, temos que

$$
P\left(X_{t}(i)=a_{i}, i \in F \mid \mathcal{F}_{t-1}\right)=\prod_{i \in F} P\left(X_{t}(i)=a_{i} \mid \mathcal{F}_{t-1}\right) .
$$

A probabilidade de ocorrer um disparo do neurônio $i$ no instante $t$ é dado por

$$
P\left(X_{t}(i)=1 \mid \mathcal{F}_{t-1}\right)=\phi\left(U_{t}^{i}, t-L_{t}^{i}\right),
$$

onde

$$
U_{t}^{(i)}=\sum_{j} W_{j \rightarrow i} \sum_{s=L_{t}^{(i)}}^{t-1} g_{j}(t-s) X_{s}(j),
$$

é o potencial da atividade acumulada dos neurônios que influênciam $i$ desde o último disparo do neurônio $i$. E $\phi_{i}: \mathbb{R} \times \mathbb{N} \rightarrow[0,1]$ e $g_{j}: \mathbb{N} \rightarrow \mathbb{R}_{+}$são funções mensuráveis para todo $i \in \mathcal{I}, j \in \mathcal{I}$.

Note que a probabilidade de um disparo em um dado neurônio $i$ é uma função que depende do potencial resultante da atividade acumulada de todos os neurônios que inflenciam $i, \mathcal{V} . \rightarrow i=\{j \in$ $\left.\mathcal{I}: W_{j \rightarrow i} \neq 0\right\}$, e do tempo decorrido desde o último disparo de $i$.

Observe que no caso em que a função $\phi_{i}$ é crescente com respeito à primeira coordenada, a contribuição dos neurônios do conjunto $\mathcal{V} . \rightarrow i$ é ou excitatória ou inibitória, dependendo do sinal de $W_{j \rightarrow i}$.

O modelo aceita que um neurônio que fica um longo período sem emitir um potencial de ação pode morrer. A segunda coordenada da função $\phi_{i}$ considera esse efeito que um longo período sem 
uma emissão de um potencial de ação tem sobre os neurônios. Queremos que quanto maior for o intervalo entre o instante $t$ e o instante do último disparo, maior seja a probabilidade de um disparo, ou seja, queremos que $\phi_{i}$ seja crescente com relação à segunda coordenada.

A função $g_{j}$ representa a perda temporal do potencial que o neurônio $i$ recebe do disparo do neurônio $j$, dizemos que $g_{j}$ é um fator de envelhecimento. Podemos pensar que a contribuição de um disparo dentro do sistema diminui ao longo do tempo, o que se traduz na hipótese de $g_{j}$ decrescente para todo $j \in \mathcal{I}$.

Na próxima seção, vamos enunciar dois teoremas de existência e unidade do processo definido nesta seção. No primeiro caso, supõe-se que o sistema permita a ocorrência de disparos espontâneos, sem assumir qualquer condição de somabilidade sobre a função $g_{j}$. Já no segundo caso, não é necessário a existência de disparos espontâneos, mas precisamos assumir que há um decaimento rápido do fator de envelhecimento $g_{j}$.

\subsection{Teorema de Existência e Unicidade}

Suponha que os pesos sinápticos são uniformementes somáveis, isto é,

$$
\sup _{i \in \mathcal{I}} \sum_{j}\left|W_{j \rightarrow i}\right|<\infty
$$

Vamos assumir que $\phi_{i}$ é uniforme Lipschitz contínuo, isto é, existe uma constante positva $\gamma$ tal que para todo $s, s^{\prime} \in \mathbb{R}, n \in \mathbb{N}, i \in \mathcal{I}$

$$
\left|\phi_{i}(s, n)-\phi_{i}\left(s^{\prime}, n\right)\right| \leq \gamma\left|s-s^{\prime}\right|
$$

Para cada neurônio $i$,

$$
\mathcal{V} \cdot \rightarrow i=\left\{j \in \mathcal{I}: j \neq i, W_{j \rightarrow i} \neq 0\right\},
$$

é o conjunto de todos os neurônios que influenciam diretamente o neurônio $i$. Note que no modelo, $\mathcal{V} \rightarrow i$ pode ser finito ou infinito.

Vamos fixar, para cada $i \in \mathcal{I}$, uma sequência crescente $\left(V_{i}(k)\right)_{k \geq-1}$ de subconjuntos de $\mathcal{I}$ tais que $V_{i}(-1)=\emptyset, V_{i}(0)=\{i\}$ e para $k \geq 1$

$$
V_{i}(k) \subset V_{i}(k+1),
$$

onde

$$
V_{i}(k) \neq V_{i}(k+1), \text { se } V_{i}(k) \neq \mathcal{V} . \rightarrow i \cup\{i\}
$$

e

$$
\bigcup_{k \geq-1} V_{i}(k)=\mathcal{V}_{\cdot \rightarrow i} \cup\{i\}
$$

Note que $\left(V_{i}(k)\right)_{k \geq-1}$ é uma sequência crescente de subconjuntos dos neurônios que influenciam $i$, incluindo o próprio neurônio $i$.

Eles consideram dois tipos de sistemas. O primeiro sistema incorpora a possibilidade de haver instantes de disparos espontâneos. Estes disparos espontâneos podem ser interpretados como estímulos externos, ou como atividade autónoma do cérebro. A existência e unicidade desta classe está garantida pelo seguinte teorema. 
Teorema 2.2.1 (Existência e unicidade para sistemas com disparos espontâneos). Sob as condições (2.4) e (2.5), assuma que as funções $\phi_{i}$ e $g_{j}$ satisfazem as seguintes suposições:

1. existe $\delta>0$ tal que para todo $i \in \mathcal{I}, s \in \mathbb{R}$ e $n \in \mathbb{N}$,

$$
\phi_{i}(s, n) \geq \delta
$$

2. temos que

$$
G(1)+\sum_{n=2}^{\infty}(1-\delta)^{n-2} n^{2} G(n)<\infty
$$

onde $G(n)=\sup _{i} \sum_{m=1}^{n} g_{i}(m)$ e onde $\delta$ é como na condição 1,

3. temos um decaimento rápido dos pesos sinápticos, isto é

$$
\sup _{i} \sum_{k \geq 1}\left|V_{i}(k)\right|\left(\sum_{j \notin V_{i}(k-1)}\left|W_{j \rightarrow i}\right|\right)<\infty .
$$

Então, sob as condições (2.6)-(2.8), existe um parâmetro $\left.\delta_{*} \in\right] 0,1\left[\right.$ tal que para $\delta>\delta_{*}$, existe uma única medida de probabilidade $P$ em $\{0,1\}^{\mathcal{I} \times \mathbb{Z}}$, sob o qual a cadeia canônica sastisfaz (2.1) e (2.2).

Note que a condição (2.6), garante a existência de disparos espontâneos, já que $\phi_{i}$ é estritamente positivo.

No sistema definido no Teorema 2.2.1, nenhuma condição de somabilidade é imposta sobre a função $g_{j}$. Em particular, a escolha $g_{j}(t)=1$ é possível. Isso implica que a especificação da cadeia é não contínua. Mais precisamente, seja

$$
p_{(i, t)}(1 \mid x)=\phi_{i}\left(U_{t}^{(i)}, t-L_{t}^{(i)}(x)\right),
$$

onde $L_{t}^{(i)}(x)=\sup \left\{s<t: x_{s}(i)=1\right\}$, temos que

$$
\sup _{x, y: x=y \text { on } V_{i}(k) \times[t-k, t-1]}\left|p_{(i, t)}(1 \mid x)-p_{(i, t)}(1 \mid y)\right| \not \supset 0 \text { quando } k \rightarrow \infty
$$

no caso em que $g_{j}(t)=1$ para todo $j$.

No segundo tipo de sistema, não assumimos uma taxa mínima de disparo. Mas adicionalmente, assumimos um decaimento suficientemente rápido do fator de envelhecimento $g_{j}$, ver condição 2.9 .

Teorema 2.2.2 (Existência e unicidade em sistemas com memória uniformemente somável). Suponha que $\phi_{i}(s, n)=\phi_{i}(s)$ não depende de $n$. Sob as condições (2.4) e (2.5). Assuma que

$$
\sup _{i} \sum_{k \geq 0}(k+1)\left|V_{i}(k)\right|\left(\sum_{j \notin V_{i}(k-1)}\left|W_{j \rightarrow i}\right| \sum_{n=1}^{\infty} g_{j}(n)+\sum_{j \in V_{i}(k-1)}\left|W_{j \rightarrow i}\right| \sum_{n=k \vee 1}^{\infty} g_{j}(n)\right)<\frac{1}{\gamma},
$$

onde $\gamma$ é dado em (2.5). Então existe uma única medida de probabilidade $P$ em $\{0,1\}^{\mathcal{I} \times \mathbb{Z}}$ tal que sob P, a cadeia canônica satisfaz (2.1) e (2.2).

A condição (2.9) implica que a especificação da cadeia é contínua. Esta é a principal diferença entre os dois sistemas considerados nos teoremas.

As provas da existência e unicidade para os dois tipos de sistemas considerados são baseadas na decomposição condicional tipo Kalikow das probabilidades de transições $\phi_{i}$, onde a decomposição é com respeito à todas as possíveis vizinhança de interação dos neurônios $i$. Para maiores detalhes ver Galves e Löcherbach (2013). 
A decomposição das probabilidades de transição podem ser interpretadas da seguinte maneira. Num primeiro passo, escolhemos aleatoriamente um alcance espacial de interação de acordo com uma distribuição de probabilidade. Uma vez que fixamos um alcance de interação, então podemos realizar uma transição de acordo com uma probabilidade que depende apenas da configuração espaço-tempo finita $x_{L_{t}^{i}}^{t-1}\left(V_{i}(k)\right)$. Uma introdução para esta técnica pode ser encontrada nas notas de aula de Fernández et al. (2001).

A decomposição tipo Kalikow implica na existência de um algoritmo de simulação perfeita da cadeia estocástica $\left(X_{t}\right)_{t \in \mathbb{Z}}$. Por algoritmo de simulação perfeita, queremos dizer que existe um algoritmo de simulação que gera como saída uma amostra com lei estacionário $P$.

\subsection{Correlação entre intervalos sucessivos entre disparos}

Vamos considerar um sistema finito com um número grande de neurônios $N$ com pesos sinápticos aleatórios $W_{i \rightarrow j}, i \neq j$.

Suponha que a sequência $\left(W_{i \rightarrow j}\right), i \neq j$, é uma sequência de variáveis aleatórias i.i.d. com distribuição Bernoulli, definidas em algum espaço de probabilidade $(\tilde{\Omega}, \tilde{\mathcal{A}}, \tilde{P})$, com parâmetro $p=$ $p_{N}$, isto é

$$
\tilde{P}\left(W_{i \rightarrow j}=1\right)=1-\tilde{P}\left(W_{i \rightarrow j}=0\right)=p_{N},
$$

onde

$$
p_{N}=\frac{\lambda}{N} \text { e } \lambda=1+\frac{\vartheta}{N} \text { para algum } 0<\vartheta<\infty .
$$

Podemos representar a sequência dos pesos sinápticos como um grafo direcionado aleatório, onde a aresta direcionada $i \rightarrow j$ existe se e somente se $W_{i \rightarrow j}=1$. Esse grafo é conhecido como grafo direcionado aleatório de Erdös-Rényi.

Assuma que $W_{j \rightarrow j} \equiv 0$ para todo $j \in \mathcal{I}$. Note que os pesos sinápticos $W_{i \rightarrow j}$ e $W_{j \rightarrow i}$ são distintos e variáveis aleatórias independentes. Condicionado na escolha das conectividades $W=\left(W_{i \rightarrow j}, i \neq j\right)$, a dinâmica do processo é dada por

$$
P^{W}\left(X_{t}(i) \mid \mathcal{F}_{t-1}\right)=\phi_{i}\left(\sum_{j} W_{j \rightarrow i} \sum_{s=L_{t}^{i}}^{t-1} g_{j}(t-s) X_{s}(j)\right)
$$

onde $P^{W}$ denota a lei condicional do processo, condicionado na escolha de $W$.

Supomos que $\phi_{i}$ é uma função que depende apenas da atividade acumulada resultante dos potenciais de ação provenientes dos neurônios que afetam o neurônio $i$.

Para um neurônio $i$ fixado, considere a sequência dos instantes de disparos sucessivos associado ao neurônio $i$

$$
\cdots<S_{-n}^{i}<\cdots<S_{0}^{i} \leq 0<S_{1}^{i}<\cdots<S_{n}^{i}<\cdots
$$

onde

$$
\begin{aligned}
S_{1}^{i} & =\inf \left\{t \geq 1: X_{t}(i)=1\right\}, \\
S_{n}^{i} & =\inf \left\{t>S_{n-1}^{i}: X_{t}(i)=1\right\} \text { para } n \geq 2
\end{aligned}
$$

e

$$
\begin{aligned}
S_{0}^{i} & =\sup \left\{t \leq 0: X_{t}(i)=1\right\}, \\
S_{-n}^{i} & =\sup \left\{t<S_{-n+1}^{i}: X_{t}(i)=1\right\} \text { para } n \geq 1 .
\end{aligned}
$$


Dado $W$ fixado, estamos interessados na covariância entre intervalos sucessivos entre disparos, para qualquer $k \neq 0,1$

$\operatorname{Cov}^{W}\left(S_{k+1}^{i}-S_{k}^{i}, S_{k}^{i}-S_{k-1}^{i}\right)=E^{W}\left[\left(S_{k+1}^{i}-S_{k}^{i}\right)\left(S_{k}^{i}-S_{k-1}^{i}\right)\right]-E^{W}\left[S_{k+1}^{i}-S_{k}^{i}\right] E^{W}\left[S_{k}^{i}-S_{k-1}^{i}\right]$,

onde $E^{W}$ denota o valor esperado condicionado na escolha de $W$.

Como o processo é estacionário, a covariância acima não depende da escolha de um $k$ particular. No próximo teorema, Galves e Löcherbach (2013), obtiveram um limitante superior explícito para a correlação entre intervalos suscessivos entre disparos, quando consideramos o sistema descrito nesta seção. Esse limitante depende do número de componentes do sistema, como também do tamanho típico de um intervalo entre disparos. Mostrando que intervalos entre disparos vizinhos são assintóticamente não correlacionados quando o número de neurônios $N$ tende ao infinito.

Teorema 2.3.1. Assuma que (2.5), (2.6) e (2.7) são satisfeitas. Então existe um subconjunto mensurável $A \in \tilde{\mathcal{A}}$, tal que em $A$,

$$
\left|\operatorname{Cov}^{W}\left(S_{3}^{i}-S_{2}^{i}, S_{2}^{i}-S_{1}^{i}\right)\right| \leq \frac{3}{\delta^{2}} N(1-\delta)^{\sqrt{N}}
$$

onde $\delta$ é o limitante inferior da condição (2.5). Mais ainda,

$$
\mathbb{P}\left(A^{c}\right) \leq e^{2 \vartheta} N^{-1 / 2} .
$$

Para $N$ grande, se o grafo dos pesos sinápticos pertence ao conjutno $A$, o resultado acima é compatível com a discussão em Gerstner e Kistler (2002). No entanto, para sistemas com um número pequeno de componentes a correlação é negativa, como apresentado em Nawrot et al. (2007). Portanto, ambas caractersticas são capturadas pelo modelo. 


\section{Capítulo 3}

\section{Modelo básico e o Teorema de Existência e Unicidade}

Neste capítulo, vamos introduzir o novo sistema de partículas com infinitos componentes interagentes que propomos nesta tese. Este é baseado no processo proposto por Galves e Löcherbach (2013) que introduzimos no Capítulo 2. Eles consideram sistemas com infinitas cadeias com memória de alcance variável interagentes, em que a probabilidade de ocorrência ou não de um disparo de um dado neurônio é uma função que da história do sistema desde o instante do último disparo do neurônio de interesse.

Como visto anteriormente no Capítulo 2, a evolução temporal desse processo é em tempo discreto. As interações entre os neurônios são definidas pela família de pesos sinápticos $\left\{W_{j \rightarrow i} ; i \in\right.$ $\mathcal{I}, j \in \mathcal{I}\}$, onde $W_{j \rightarrow i} \in \mathbb{R}$, esta característica permite que o processo considere dois tipos de estímulo, excitatório e inibitório, dependendo do sinal de $W_{j \rightarrow i}$.

No nosso caso, consideramos um processo em tempo contínuo. As interações entre os neurônios são definidas por grafos orientados que dependem da intensidade do potencial de membrana. Não vamos considerar o fator de envelhecimento dos estímulos no nosso modelo.

Vamos considerar o caso em que cada neurônio pode apenas estimular a ocorrência de um disparo em outros neurônios, isto é, os pesos sinápticos são sempre iguais a 1, se existe uma ligação entre os neurônios e 0 caso contrário.

Biologicamente, o modelo representa a dinâmica do potencial de membrana de um conjunto enumerável de neurônios. O potencial de ação é representado no modelo pela perda total do potencial de membrana. Ou seja, quando ocorre um potencial de ação em algum neurônio do sistema, o potencial de membrana deste neurônio imediatamente vai para zero, no intervalo de tempo entre o instante de ocorrência de um potencial de ação até o instante em que o neurônio recebe cargas positivas, por estímulos externos ou pelo estímlo proveniente de outros neurônios, dizemos que o neurônio se encontra num estado de repouso.

Neste capítulo, vamos enunciar também o primeiro resultado que garante a existência e unicidade do modelo proposto. No final da seção apresentamos dois exemplos de processos que satisfazem as condições do teorema de existência e unicidade.

\subsection{Definições}

Seja $\mathcal{I}$ um conjunto enumerável de neurônios. Denotamos por $\mathcal{S}=\mathbb{N}^{\mathcal{I}}$ o espaço das configurações. Os elementos de $\mathcal{S}$ são configurações denotadas pelas letras gregas $\eta, \zeta, \xi, \ldots$ A configuração do sistema no instante $t \in \mathbb{R}$ é denotada por

$$
\xi_{t}=\left(\xi_{t}(i), i \in \mathcal{I}\right)
$$

onde $\xi_{t}(i) \in \mathbb{N}$, para cada neurônio $i \in \mathcal{I}$, é o valor do potencial de membrana do neurônio $i$ no instante $t$. 


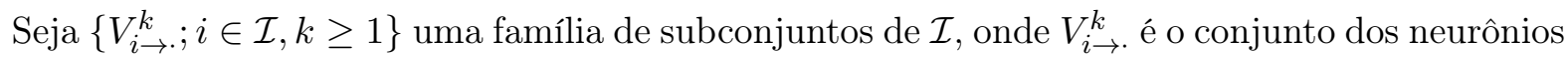
que são afetados pelo potencial de ação do neurônio $i$, quando o potencial de membrana do neurônio

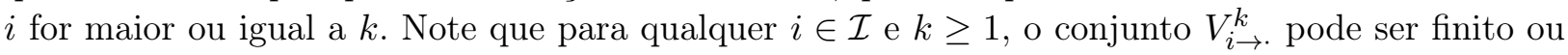
infinito.

Para definir a dinâmica do processo $\left(\xi_{t}\right)_{t \in \mathbb{R}}$, precisamos definir a família de transformações $\left\{\pi^{i, k}: i \in \mathcal{I}, k \geq 0\right\}$. Para cada $i \in \mathcal{I}$,

$$
\pi^{i, k}(\xi)(u)= \begin{cases}\xi(u) & \text { se } \xi(i)<k \\ \xi(u) & \text { se } u \neq i, u \notin V_{i \rightarrow}^{k} \text {. e } \xi(i) \geq k \\ \xi(u)+1 & \text { se } u \neq i, u \in V_{i \rightarrow}^{k} \text { e } \xi(i) \geq k \\ 0 & \text { se } u=i \text { e } \xi(i) \geq k\end{cases}
$$

para $k \geq 1$.

Para $k=0$

$$
\pi^{i, 0}(\xi)(u)= \begin{cases}\xi(u) & \text { se } u \neq i \\ \xi(u)+1 & \text { se } u=i\end{cases}
$$

Em outras palavras, dada uma configuração $\xi \in \mathcal{S}$, a transformação $\pi^{i, k}$ apenas alterará a configuração $\xi$ quando o potencial de membrana do neurônio $i$ for igual ou maior que o valor $k$ definida na transformação, isto é, se $\xi(i) \geq k$. Se $\xi(i)<k$, dizemos que o neurônio $i$ não possue potencial de membrana suficiente para que ocorra um potencial de ação. Essa dinâmica caracteriza o potencial de ação de um neurônio, o valor $k$ da transformação representa o limiar excitatório da membrana, consideramos que este limiar é variável.

No caso em que ocorre um potencial de ação em $i$, o neurônio $i$ perde todo o seu potencial de membrana voltando para o estado de repouso. Simultanêamente, os potenciais de membrana dos neurônios dentro do conjunto $V_{i \rightarrow \text { }}^{k}$. são alteradas, pela adição de uma unidade no potencial de membrana de cada neurônio.

O estímulo externo é representado pelas tranformações $\pi^{i, 0}$, para $i \in \mathcal{I}$. O estímulo externo não causa um disparo imediato nos neurônios mas aumenta as chances de um disparo, aumentando o potencial de membrana do neurônio.

Vamos assumir que $i \notin V_{i \rightarrow \text {. }}^{k}$, para todo $i \in \mathcal{I}$ e $k \geq 1$. Isto implica que a atividade do neurônio $i$ não influencia sua própria configuração de maneira imediata. Isso não excluí a possibilidade de que um potencial de ação emitido por um neurônio possa influenciar a sua própria configuração depois do instante em que ocorreu o potencial de ação.

Como todo o potencial de ação que ocorre dentro do sistema estímula a ocorrência de outros potenciais de ação, dependendo da família de conjuntos $\left\{V_{i \rightarrow \text {. }}^{k}: i \in \mathcal{I}, k \geq 1\right\}$, existe a possibilidade de que o estímulo gerado por um neurônio se propague para outros neurônios e que eventualmente volte para o neurônio inicial.

Na Figura 3.1, apresentamos um exemplo onde o estímulo gerado pelo potencial de ação do neurônio $i$ se propaga pelo sistema e retorna ao próprio neurônio $i$. No exemplo ilustramos o caso em que o potencial de ação gerado pelo neurônio $i$ estímula outros dois neurônios.

O estímulo gerado pelo potencial de ação do neurônio $i$ no instante $t_{1}$ é fundamental para que ocorra um potencial de ação em $j$ no instante $t_{2}$. Estes dois potenciais de ação, acrescentam duas unidades ao potencial de membrana de $u$, tornando possível o potencial de ação do neurônio $u$ no instante $t_{3}$. Finalmente, no instante $t_{3}$, o estímulo gerado em $t_{1}$ retorna ao neurônio $i$.

Podemos observar que, para $k$ fixado, os conjuntos $\left\{V_{i \rightarrow .}^{k}\right\}_{i \in \mathcal{I}}$ definem um grafo orientado, onde $\mathcal{I}$ é o conjunto de vértices e a aresta orientada de $i$ para $j, i \rightarrow j$, existe se e somente se $j \in V_{i \rightarrow \text {. }}^{k}$. Note que para diferentes valores de $k$ temos também diferentes grafos de interação. No processo, a evolução temporal das interações entre os neurônios é dada pelos diferentes grafos orientados definidos pelos conjuntos $\left\{V_{i \rightarrow \text {. }}^{k}\right\}_{i, k}$.

Agora podemos definir a dinâmica do processo. Vamos considerar um sistema markoviano de partículas em $\mathcal{S}$ e com gerador infinitesimal $\mathcal{L}$. Para qualquer função cilíndrica limitada $f: \mathcal{S} \rightarrow \mathbb{R}$, 


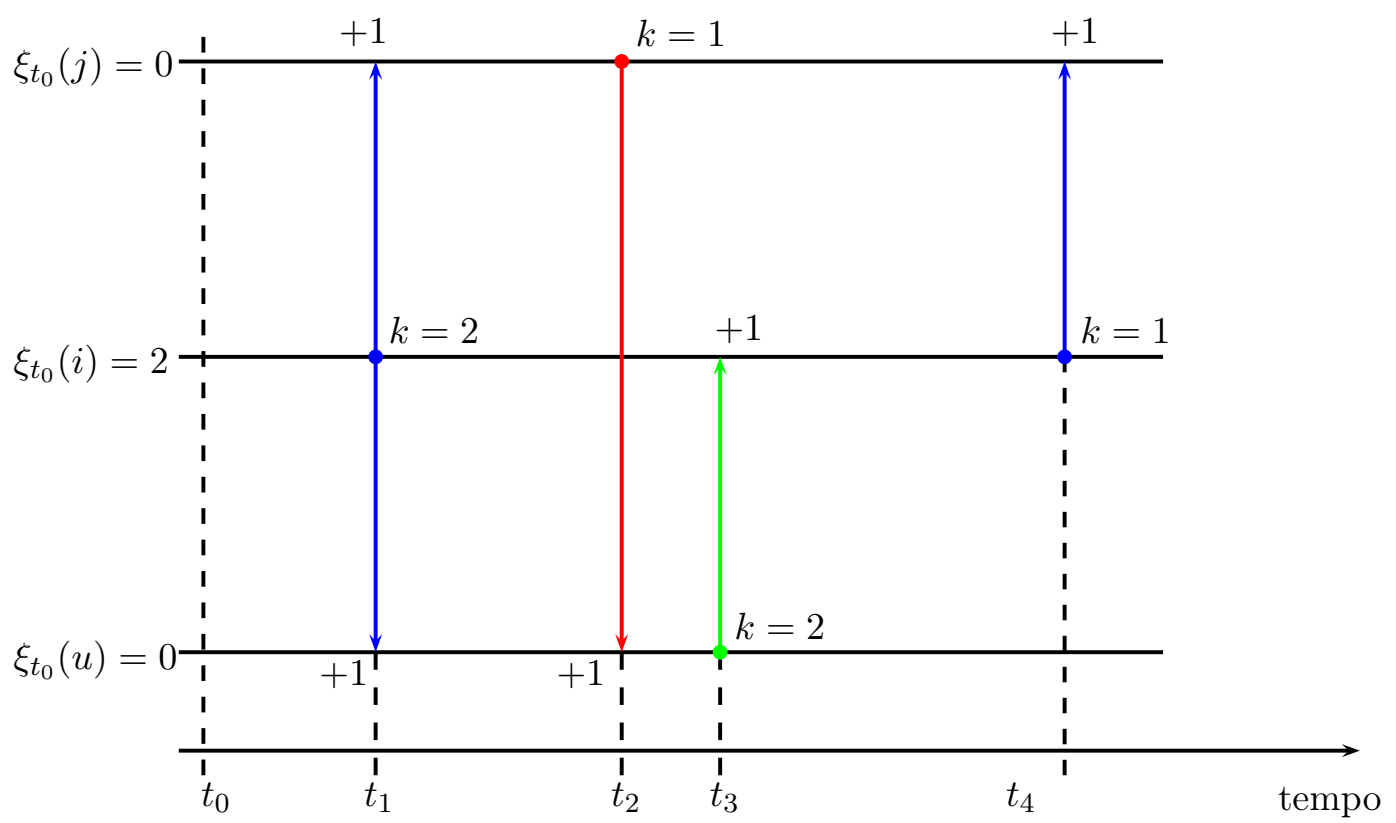

Figura 3.1: Exemplo de retorno de estímulo

o gerador $\mathcal{L}$ é dado por

$$
\mathcal{L} f(\xi)=\sum_{i \in \mathcal{I}} \sum_{k \geq 0} \lambda_{i}(k)\left[f\left(\pi^{i, k}(\xi)\right)-f(\xi)\right]
$$

onde, para cada $i \in \mathcal{I}, \lambda_{i}: \mathbb{N} \rightarrow \mathbb{R}_{+}$.

Note que $\lambda_{i}(k), i \in I$ e $k \geq 0$, é a taxa de ocorrência da transformação $\pi^{i, k}$. Para cada $i \in \mathcal{I}$, podemos definir a seguinte quantidade

$$
\Lambda_{i}=\lambda_{i}(0)+\sum_{k \geq 1} \lambda_{i}(k)+\sum_{k \geq 1} \sum_{j \in V_{\rightarrow \rightarrow i}^{k}} \lambda_{j}(k)
$$

onde $V_{. \rightarrow i}^{k}=\left\{j \in \mathcal{I}: i \in V_{j \rightarrow \text {. }}^{k}\right\}$ é o conjunto de neurônios que podem influenciar o neurônio $i$. Logo, $\Lambda_{i}$ é a taxa de ocorrência de todas as transformações que podem mudar o potencial de membrana do neurônio $i$. Lembrando que, para $j \in V_{. \rightarrow i}^{k}$, a transformação $\pi^{j, k}$ afeta o valor de $\xi(i)$ se e somente se $\xi(j) \geq k$.

As transformações $\left\{\pi^{i, 0}\right\}_{i \in \mathcal{I}}$ são as únicas que alteram apenas o valor do potencial de membrana do neurônio $i$, mantendo o restante do sistema inalterado. Para cada $i \in \mathcal{I}$, defina

$$
\rho_{i}=\frac{\lambda_{i}(0)}{\sum_{k \geq 0} \lambda_{i}(k)},
$$

$\rho_{i}$ é a probabilidade de $k=0$ dado que ocorreu uma transformação $\pi^{i, k}$. 


\subsection{Teorema de Existência e Unicidade}

Uma pergunta natural é se existe pelo menos um processo estacionário que é consistente com gerador infinitesimal $\mathcal{L}$ definido em (3.1), e se sim, se esse processo é único. Nosso primeiro resultado é o teorema de existência e unicidade do processo introduzido na Seção 3.1. Nele mostramos que, sob algumas hipóteses para a família de funções $\left\{\lambda_{i}(\cdot), i \in \mathcal{I}\right\}$.

Teorema 3.2.1. Suponha que $\left\{\lambda_{i}(k) ; i \in \mathcal{I}, k \geq 0\right\}$ satisfaça as seguintes hipóteses:

1.

$$
\beta=\sup _{i \in \mathcal{I}} \Lambda_{i}<\infty
$$

2. Para todo $i \in \mathcal{I}$,

$$
\sum_{k \geq 1} \lambda_{i}(k)\left(\rho_{i}\right)^{k}-\sum_{k \geq 1} \sum_{j \in V_{: \rightarrow i}^{k}} \lambda_{j}(k) \geq 0 .
$$

Então existe um único processo estacionário $\left(\xi_{t}\right)$ assumindo valores em $\mathcal{S}$, com trajetória contínua $\grave{a}$ direita com limites à esquerda e com gerador infinitesimal $\mathcal{L}$ dado por (3.1).

A prova do Teorema 3.2.1 será dada no Capítulo 6. Para provar a existência e unicidade do Teorema 3.2.1, usamos a técnica de simulação perfeita.

\subsection{Exemplo}

A condição (3.5) do Teorema 3.2.1 é bastante restritiva. Nesta seção, vamos apresentar um exemplo onde as condições do Teorema 3.2.1 são satisfeitas.

Seja $s: \mathbb{N} \rightarrow \mathbb{R}_{+}$uma função positiva tal que

$$
\sum_{k \geq 0} s(k)<+\infty
$$

Para cada $i \in \mathcal{I}$, defina

$$
\lambda_{i}(k)=a_{i} s(k) \text { para } k \geq 0,
$$

onde $a_{i}>0$.

Suponha que

$$
\sup \left\{\bar{a}_{i}: i \in \mathcal{I}\right\}<+\infty
$$

onde $\bar{a}_{i}=\sup \left\{a_{j}: j \in \bigcup_{k \geq 1} V_{\cdot \rightarrow i}^{k}\right\}$.

Vamo supor que a cardinalidade dos conjuntos $\left\{V_{\cdot \rightarrow i}^{k} ; i \in \mathcal{I}, k \geq 1\right\}$ é limitada por $c_{k} \in \mathbb{N}$ que depende apenas de $k$. Isto é, para cada $i \in \mathcal{I}$ e $k \geq 1$, temos que

$$
\left|V_{\cdot \rightarrow i}^{k}\right| \leq c_{k},
$$

onde $|\cdot|$ denota a cardinalidade do conjunto.

Para $i \in \mathcal{I}$ fixado, temos que

$$
\begin{aligned}
\Lambda_{i} & =a_{i} \sum_{k \geq 0} s(k)+\sum k \geq 1 \sum_{j \in V_{\cdot \rightarrow i}^{k}} a_{j} s(k) \\
& \leq a_{i} \sum_{k \geq 0} s(k)+\bar{a}_{i} \sum_{k \geq 1} c_{k} s(k) .
\end{aligned}
$$


Observe que

$$
\sum_{k \geq 1} \sum_{j \in V_{\cdot \rightarrow i}^{k}} \lambda_{j}(k) \leq \bar{a}_{i} \sum_{k \geq 1} s(k) c_{k}
$$

$\mathrm{e}$

$$
\sum_{k \geq 1} \lambda_{i}(k)\left(\rho_{i}\right)^{k}=a_{i} \sum_{k \geq 1} s(k) \rho^{k}
$$

onde $\rho=\frac{s(0)}{\sum_{k \geq 0} s(k)}$ é uma constante que não depende do neurônio $i$.

Portanto, para que as condições (3.4) e (3.5) sejam satisfeitas, precisamos que

$$
\sum_{k \geq 1} c_{k} s(k)<+\infty
$$

$\mathrm{e}$

$$
\frac{\bar{a}_{i}}{a_{i}} \leq \frac{\sum_{k \geq 1} s(k) \rho^{k}}{\sum_{k \geq 1} c_{k} s(k)}
$$

para todo $i \in \mathcal{I}$.

Como $\sum_{k>0} s(k)<\infty$, então uma condição suficiente para que (3.6) seja satisfeita, é que $c_{k}$ seja uma sucessão decrescente com limite zero. Mas essa condição não é necessária. Com efeito suponha que $s(k)=\frac{1}{k !}$, então se $c_{k}=d^{k}$ temos que

$$
\sum_{k \geq 1} \frac{d^{k}}{k !}=e^{d}-1
$$

para qualquer $d \in \mathbb{R}_{+}$e a condição (3.6) é satisfeita. Nestas condições, temos que o número de neurônios, cuja a atividade influenciam o neurônio $i$, para todo $i \in \mathcal{I}$, cresce exponencialmente em relação ao potencial de membrana $k$. Porém, a probabilidade de ocorrer uma transformações com potencial de membrana alta decresce com relação a $k$.

Vamos considerar que a sequência $\left(c_{k}\right)_{k \geq 1}$ e a função $s(\cdot)$ satisfazem a condição (3.6). Como $\rho \in(0,1)$, então pela equação $(3.7)$ precisamos que a sequência $\left(a_{i}\right)_{i \in \mathcal{I}}$ satisfaça, para todo $i \in \mathcal{I}$, a seguinte desigualdade

$$
a_{i}>r \bar{a}_{i}
$$

onde $r$ é um interio positivo.

Seja $\mathcal{I}=\{1,2, \ldots\}$ o conjunto dos neurônios. Vamos considerar os seguintes grafos de interações

$$
V_{i \rightarrow .}^{k}=\bigcup_{j=i+1}^{k+i}\{j\}, \text { para } \mathrm{i} \in \mathcal{I} \text { e } \mathrm{k} \geq 1 .
$$

Para um neurônio $i \in \mathcal{I}$ fixado, observe que $i$ recebe influência apenas dos neurônios $\{1, \ldots, i-$ $1\}$. Note também que o neurônio 1 não é influenciado por outros neurônios. Em outras palavras, temos que

$$
V_{1}^{k}=\emptyset, \text { para todo } k \geq 1 \text {, }
$$




$$
V_{\cdot \rightarrow i}^{k}=\bigcup_{j=i-k}^{i-1}\{j\} \text { para } k<i
$$

e

$$
V_{\cdot \rightarrow i}^{k}=V_{\cdot \rightarrow i}^{i-1} \text { para } k \geq i .
$$

Portanto, se considerarmos a sequência

$$
a_{i}=r a_{i-1}+1 \text { para } i \geq 2,
$$

a condição (3.8) é satisfeita. 


\section{Capítulo 4}

\section{Uma algoritmo de simulação perfeita para o processo $\left(\xi_{t}\right)_{t \in \mathbb{R}}$}

Neste capítulo, vamos apresentar o esquema de simulação perfeita para o processo introduzido no Capítulo 3. A idéia é desenvolver um método que encontra, para todo neurônio $i \in \mathcal{I}$, o valor $\xi_{t}(i)$ no instante $t \in \mathbb{R}$.

Na primeira seção introduzimos o conceito de simulação perfeita. Em seguida, vamos definir para todo $i \in \mathcal{I}$ o processo rascunho para trás que descreve a história dos neurônios que podem eventualmente influenciar o valor do potencial de membrana do neurônio $i$. Na última seção apresentamos o pseudo-algoritmo para a realização da simulação perfeita, como também o resultado que garante que o número de passos do algoritmo é finito quase certamente.

\subsection{Simulação perfeita}

Simulação perfeita é o nome dado para qualquer algoritmo que gere como saída uma amostra de um processo estacionário cuja distribuição é garantida que segue uma dada lei de probabilidade. Pode se mostrar que quando é possível produzir tal amostra, então o processo existe, por construção, e é único.

Propp e Wilson (1995) foram os primeiros a desenvolverem um método para a realização de uma simulação perfeita. Neste artigo eles introduzem o algoritmo "acoplamento desde o passado" (Coupling from the past, em inglês), para processos de Markov com tempo finito de coalescência. Isto é, de processos para os quais existem um acoplamento entre as trajetórias tais que, com probabilidade 1 as trajetórias começando de qualquer condição inicial coalescem em um tempo finito. Isto inclui todos os processos irredutíveis de Markov com espaço de estado finito.

A ideia de Propp e Wilson (1995) foi generalizada por Comets et al. (2002) no caso das cadeias de alcance infinito, utilizando uma construção baseada em um esquema regenerativo. Dizemos que uma cadeia de alcance infinito tem um esquema regenerativo quando é possível gerar a cadeia usando os instantes de um processo de renovação auxiliar que divide a sequência em sucessivos blocos independentes. A noção de simulação pefeita está relacionada à noção de esquema regenerativo, pois os pontos de regeneração podem ser utilizados como ponto de partida para a construção da cadeia.

Fernández et al. (2002) introduziram outro esquema de simulação perfeita, o algoritmo "rascunho para trás" (Backward-Forward, em inglês). Este esquema é aplicável, em princípio, para qualquer processo que pode ser amostrado a partir de um processo espacial de nascimento e morte e que seja contínuo com respeito a um processo pontual de Poisson.

Este esquema de simulação perfeita não envolve o acoplamento dos processos com diferentes condições iniciais. O algoritmo é baseado em um procedimento de duas etapas: (i) na primeira etapa definimos um esquema de simulação perfeita para gerar o conjunto dos "ancestrais", isto é, dos antecessores que podem influenciar o valor de $\xi_{0}(i)$, e (ii) na segunda etapa, usando as informações da primeira etapa, a amostra é gerada de acordo com as regras do processo de interesse. A segunda 
etapa está condicionada ao sucesso da primeira etapa, isto é, se é possível gerar em tempo finito o conjunto dos ancestrais.

Baseado no procedimento rascunho para trás, Galves et al. (2010) abordaram o problema da simulação perfeita para a medida de Gibbs com interações de alcance infinito. Em Galves et al. (2013), eles consideram um sistema de partícula em $\mathbb{Z}^{d}$ com espaço de estados em $\mathbb{R}$ e interações de alcance infinito. Assumindo que as taxas de mudanças são contínuas, eles obtiveram uma decomposição de Kalikow para as taxas de alcance infinito. Assim, as taxas de alcance infinito são representadas através de uma mistura de taxas de alcance finito. Como uma aplicação da decomposição eles introduziram um algoritmo de simulação perfeita para o sistema.

A mesma técnica de decomposição é utilizada em Galves e Löcherbach (2013). Eles introduziram uma decomposição de Kalikow para as propabilidades de transições com ordem infinita.

No nosso caso, não precisamos usar a técnica de decomposição de Kalikow, já que as taxas das transformações do sistema não depende da configuração global do sistema. A técnica utilizada aqui é inspirada na construção de processos markovianos de saltos apresentada em Bertein e Galves (1977).

\subsection{Processo rascunho para trás}

Nosso objetivo é introduzir um algoritmo de simulação perfeita que gera como saída o valor $\xi_{t}(i)$, para $i \in \mathcal{I}$ e $t \in \mathbb{R}$ fixados. Para isso, desenvolvemos um procedimento de simulação dividida em duas etapas.

A primeira etapa do algoritmo de simulação perfeita é baseado na construção do processo rascunho para trás. Nesta primeira etapa, determinamos os ancestrais do neurônio $i$ no instante $t$. Isto é, os neurônios cuja atividades podem eventualmente influenciar o valor do potencial de membrana do neurônio $i$ no instante $t$. O conjunto destes neurônios é chamado de conjunto dos ancestrais.

Formalmente, vamos construir, para um neurônio $i \in \mathcal{I}$ fixado, o processo $\left(C_{s}^{(i)}\right)_{s \geq 0}$ começando de $\{i\}$ no instante $t$ assumindo valores em $\mathcal{P}(\mathcal{I})$, conjunto dos subconjuntos de $\mathcal{I}$, tal que $C_{s}^{(i)}$ é o conjunto dos neurônios, até o instante $-s$, cuja atividade podem eventualmente influenciar o neurônio $i$ no instante $t$. Chamamos $\left(C_{s}^{(i)}\right)_{s \geq 0}$ de processo rascunho para trás. Sem perda de generalidade, vamos considerar o caso em que $t=0$.

Vamos inicialmente descrever este procedimento de maneira informal. A ideia é construir uma sequência de sucessivos conjuntos de neurônios que podem eventualmente influenciar o valor do potencial de membrana do neurônio $i$ no instante $t=0$. Para isso, precisamos observar o que aconteceu no passado para determinar quais são os neurônios que devemos considerar no conjunto dos ancestrais.

Para $i \in \mathcal{I}$, defina

$$
C_{0}^{(i)}=\{i\}
$$

Inicialmente, precisamos observar apenas o que ocorre no passado do neurônio $i$. Observamos o passado do neurônio $i$ até encontrarmos a primeira ocorrência de uma transformação que possa alterar o valor de $\xi_{0}(i)$. Neste instante existem três possíveis eventos:

1. Acrescentar um neurônio ao conjunto dos ancestrais: esse evento ocorre quando uma transformação $\pi^{j, k}$, tal que $j \in V_{. \rightarrow i}^{k}$, for sorteada para algum $k \geq 1$ e $j \in \mathcal{I}$. Condicionada à ocorrência de uma transformação que afeta $i$, a probabilidade deste evento é dada por

$$
\frac{\sum_{k \geq 1} \sum_{j \in \mathcal{I}} \lambda_{j}(k) \mathbb{I}\left\{j \in V_{\cdot \rightarrow i}^{k}\right\}}{\Lambda_{i}} .
$$

2. Retirar o neurônio $i$ do conjunto dos ancestrais: Isso ocorre, se uma transformação $\pi^{i, k}$ for sorteada, para algum $k \geq 1$, e em seguida, para trás no tempo, forem sorteadas $k$ transformações $\pi^{i, 0}$ e no intervalo entre a ocorrência da transformação $\pi^{i, k}$ e da $k$-ésima transformação $\pi^{i, 0}$, nenhuma transformação $\pi^{i, l}$ seja sorteada, para qualquer $l \geq 1$. Então, temos 
que $\xi_{0}(i)=0$ e a partir do instante da ocorrência da $k$-ésima transformação $\pi^{i, 0}$, o potencial de membrana do neurônio $i$, no instante $t=0$, independe do restante do passado. Portanto, neste instante podemos retirar o neurônio $i$ do conjunto dos ancestrais. Condicionada à ocorrência de uma transformação que afeta $i$, a probabilidade deste evento é dada por

$$
\frac{\sum_{k \geq 1} \lambda_{i}(k)\left(\rho_{i}\right)^{k}}{\Lambda_{i}}
$$

onde $\rho_{i}=\frac{\lambda_{i}(0)}{\sum_{k \geq 1} \lambda_{i}(k)}$ definido em (3.3).

3. Manter o conjunto dos ancestrais: não alteramos o conjuntos dos ancestrais se a transformação $\pi^{i, 0}$ for sorteada. Ou se uma transformação $\pi^{i, k}$ for sorteada, para algum $k \geq 1$, mas este sorteio não é seguido por $k$ sorteios da transformação $\pi^{i, 0}$, antes de sortearmos uma transformação $\pi^{i, l}$, para qualquer $l \geq 1$. Condicionada à ocorrência de uma transformação que afeta $i$, a probabilidade deste evento é dada por

$$
\frac{\lambda_{i}(0)+\sum_{k \geq 1} \lambda_{i}(k)\left(1-\left(\rho_{i}\right)^{k}\right)}{\Lambda_{i}} .
$$

Suponha que $t_{1} \in \mathbb{R}_{+}$é o primeiro instante, após o instante $t=0$, em que ocorre uma transformação que pode afetar o neurônio $i$. Se no instante $t_{1}$ o evento sorteado foi o primeiro evento descrito acima. Então, $C_{t_{1}}^{(i)}=\{i, j\}$ e a partir desse instante precisamos observar também o passado do neurônio $j$. Se no instante $t_{1}$ observamos a ocorrência do segundo evento, então $C_{t_{1}}^{(i)}=\emptyset$. Em qualquer outro caso, o conjunto dos ancestrais continua inalterado $C_{t_{1}}^{(i)}=\{i\}$.

Repetimos esse procedimento até o instante em que $C_{s}^{(i)}=\emptyset$. Isso acontece quando observamos o segundo evento descrito para cada neurônio do conjunto dos ancestrais .

Resumindo, podemos descrever a construção do processo $\left(C_{s}^{(i)}\right)_{s \geq 0}$ da seguinte maneira:

1. Defina $C_{0}^{(i)}=\{i\}$.

2. Dado que $C_{s}^{(i)}=C, C$ subconjunto finito de $\mathcal{I}$, esperamos um tempo exponencial de parâmetro $\sum_{u \in C} \Lambda_{u}$.

3. Depois desse tempo pulamos para um conjunto finito $D \in \mathcal{P}(\mathcal{I})$ com distribuição de probabilidade dada por

$$
P(D \mid C)= \begin{cases}\frac{\sum_{k \geq 1} \lambda_{j}(k) \mathbb{I}\left\{j \in V_{\cdot \rightarrow C}^{k}\right\}}{\sum_{u \in C} \Lambda_{u}}, & \text { se } D=C \cup\{j\} \\ \frac{\sum_{k \geq 1} \lambda_{j}(k)\left(\rho_{j}\right)^{k} \mathbb{I}\{j \in C\}}{\sum_{u \in C} \Lambda_{u}}, & \text { se } D=C /\{j\} \\ \frac{\sum_{j \in C} \lambda_{j}(0)+\sum_{j \in C} \sum_{k \geq 1} \lambda_{j}(k)\left(1-\left(\rho_{j}\right)^{k}\right)}{\sum_{u \in C} \Lambda_{u}}, & \text { se } D=C \\ 0, & \text { caso contrário }\end{cases}
$$

onde $V_{\cdot \rightarrow C}^{k}=\bigcup_{u \in C} V_{\cdot \rightarrow u}^{k}$.

Agora vamos definir formalmente o processo $\left(C_{s}^{(i)}\right)_{s \geq 0}$. Denote por $\mathcal{P}_{f}(\mathcal{I})$ o conjunto dos subconjuntos finitos de $\mathcal{I}$.

Seja $\Sigma=\left\{\sigma_{i}^{(j, k)}: i, j \in \mathcal{I}, k \geq 0\right\}$ uma família de transformações em $\mathcal{P}(\mathcal{I})$, definidas da seguinte maneira. Para qualquer conjunto unitário $\{u\}$, para $j \neq i$ e $k \geq 1$,

$$
\sigma_{i}^{(j, k)}(\{u\})= \begin{cases}\{i, j\}, & \text { se } u=i \text { e } j \in V_{\cdot \rightarrow i}^{k} \\ \{u\}, & \text { caso contrário. }\end{cases}
$$


Para $j=i$ e $k \geq 1$,

$$
\sigma_{i}^{(i, k)}(\{u\})= \begin{cases}\emptyset, & \text { se } u=i \\ \{u\}, & \text { caso contrário. }\end{cases}
$$

Para $j \in \mathcal{I}$ e $k=0$,

$$
\sigma_{j}^{(j, 0)}(\{u\})=\{u\}, \text { para todo } u .
$$

Para qualquer conjunto $C \in \mathcal{P}_{f}(\mathcal{I})$, defina de forma semelhante

$$
\sigma_{i}^{(j, k)}(C)=\bigcup_{u \in C} \sigma_{i}^{(j, k)}(\{u\})
$$

Seja $\nu: \Sigma \rightarrow \mathbb{R}_{+}$uma função definida da seguinte maneira. Para $i \in \mathcal{I}$ e $k \geq 1$,

$$
\nu\left(\sigma_{i}^{(j, k)}\right)= \begin{cases}\lambda_{j}(k), & \text { se } j \neq i \text { e } j \in V_{. \rightarrow i}^{k} \\ \lambda_{i}(k)\left(\rho_{i}\right)^{k}, & \text { se } j=i \text { e } k \geq 1 \\ \lambda_{i}(0)+\sum_{l \geq 1} \lambda_{i}(l)\left(1-\left(\rho_{i}\right)^{l}\right), & \text { se } j=i \text { e } k=0 \\ 0, & \text { caso contrário. }\end{cases}
$$

O gerador infinitesimal do processo $\left(C_{s}^{(i)}\right)_{s \geq 0}$ assumindo valores em $\mathcal{P}_{f}(\mathcal{I})$, com condição incial $C_{0}^{(i)}=\{i\}$, é dado por

$$
\mathcal{G} f(C)=\sum_{\sigma \in \Sigma} \nu(\sigma)[f(\sigma(C))-f(C)]
$$

onde $f: \mathcal{P}_{f}(\mathcal{I}) \rightarrow \mathbb{R}$ é qualquer função cilíndrica limitada e $C \in \mathcal{P}_{f}(\mathcal{I})$.

A próxima proposição resume as propriedades da família $\left\{C_{s}^{(i)} ; i \in \mathcal{I}, s \in \mathbb{R}_{+}\right\}$dos processos rascunho para trás.

Proposição 4.2.1. 1. Suponha que

$$
\sum_{\sigma \in \Sigma_{i}} \nu(\sigma)<\infty
$$

para todo $i \in \mathcal{I}$, onde $\Sigma_{i}=\left\{\sigma_{i}^{(j, k)}: k \geq 0, j \in V_{. \rightarrow i}^{k} \cup\{i\}\right\}$. Então, existe um espaço de probabilidade $(\Omega, \mathcal{B}, P)$ sobre o qual podemos construir, para todo $i \in \mathcal{I}$, uma versão do processo markoviano de salto $\left(C_{s}^{(i)}, s \in \mathbb{R}_{+}\right)$em $\mathcal{P}_{f}(\mathcal{I})$, com condição inicial $\{i\}$ e com gerador infinitesimal $\mathcal{G}$ definido em (4.7).

2. Suponha também que

$$
\sup _{i \in \mathcal{I}} \sum_{\sigma \in \Sigma} \nu(\sigma)(|\sigma(\{i\})|-1)=c<\infty
$$

então, para todo $i \in \mathcal{I}$, o processo $\left(C_{s}^{(i)}\right)$ não explode. Isto é, $\left|C_{s}^{(i)}\right| \leq e^{c s}$ para qualquer $s \geq 0$.

Podemos reescrever a condição (4.8) da seguinte maneira

$$
\sum_{\sigma \in \Sigma_{i}} \nu(\sigma)=\lambda_{i}(0)+\sum_{k \geq 1} \lambda_{i}(k)\left(1-\left(\rho_{i}\right)^{k}\right)+\sum_{k \geq 1} \lambda_{i}(k)\left(\rho_{i}\right)^{k}+\sum_{k \geq 1} \sum_{j \in V_{\cdot \rightarrow i}^{k}} \lambda_{j}(k)=\Lambda_{i} .
$$


Também podemos reescrever a condição (4.9) por

$$
\sup _{i \in \mathcal{I}} \sum_{k \geq 1} \sum_{j \in V_{\cdot \rightarrow i}^{k}} \lambda_{j}(k)-\sum_{k \geq 1} \lambda_{i}(k)\left(\rho_{i}\right)^{k}=c,
$$

pela condição (3.5) do Teorema 3.2.1 temos que $c<0$.

A prova da Proposição 4.2.1 é baseada na demonstração do Teorema 1 em Bertein e Galves (1977). Sob algumas condições, eles mostraram que existe um espaço de probabilidade no qual podemos construir um processo markoviano de saltos com espaço de estados em $\mathcal{P}(S)$, onde $S$ é um conjunto enumerável. Mais ainda, mostraram também que o processo não explode.

Para mostrar a primeira parte da proposição, vamos construir o processo de saltos $\left(C_{s}^{(i)}\right)$, para todo $i \in \mathcal{I}$, através dos sucessivos tempos de saltos

$$
0<T_{1}^{(i)}<T_{2}^{(i)}<\ldots
$$

e pelos sucessivos estados $\tilde{C}_{0}^{(i)}, \tilde{C}_{1}^{(i)}, \ldots$ tais que

$$
C_{t}^{(i)}=\tilde{C}_{n}^{(i)} \quad \text { se } T_{n}^{(i)} \leq t<T_{n+1}^{(i)},
$$

com as convenções $T_{0}^{(i)}=0$ e $\tilde{C}_{0}^{(i)}=\{i\}$. Denote por $\mathcal{F}_{t}^{(i)}, t \in \mathbb{R}_{+}$, a $\sigma$-álgebra associada ao processo de $\operatorname{saltos}\left(C_{s}^{(i)}\right)$.

As sequências $\left(T_{n}^{(i)}\right)$ e $\left(\tilde{C}_{n}^{(i)}\right)$ serão construídas de modo que

1. Condicionado à $\mathcal{F}_{T_{n}^{(i)}}$, as variáveis aleatórias $T_{n+1}^{(i)}-T_{n}^{(i)}$ são exponencialmente distribuídas com parâmetro $\sum_{u \in \tilde{C}_{n}^{(i)}} \Lambda_{u}$ e independentes.

2. A sequência $\left(\tilde{C}_{n}^{(i)}\right)$ é uma cadeia de Markov com estado inicial $\{i\}$ e matriz de transição $Q$ dada por

$$
Q(C, D)=\frac{\sum_{\sigma: \sigma(C)=D} \nu(\sigma)}{\sum_{\sigma \in \Sigma_{C}} \nu(\sigma)},
$$

onde $D \in \mathcal{P}_{f}(\mathcal{I})$ e $\Sigma_{C}=\left\{\sigma_{u}^{(j, k)}: u \in C, k \geq 0, j \in V_{\cdot \rightarrow u}^{k} \cup\{u\}\right\}$.

Note que as probabilidades de transição definidas pela matriz $Q$ são equivalentes as probabilidades definidas em (4.1).

Demonstração. Prova da primeira parte: Seja $\left\{N^{\sigma}, \sigma \in \Sigma\right\}$ uma família de processos pontuais de Poisson em $\mathbb{R}_{+}$independentes, com parâmetro $\nu(\sigma)$, respectivamente, definidas em um espaço de probabilidade $(\Omega, \mathcal{B}, P)$.

Vamos introduzir o processo pontual de Poisson $N$, definido em $] 0,+\infty[\times \Sigma$, provido da $\sigma$-álgebra produto das $\sigma$-álgebra de Borel e a $\sigma$-álgebra das partes de $\Sigma$, da seguinte maneira

$$
N(B \times I)=\sum_{\sigma \in I} N^{\sigma}(B),
$$

para todo $B \in \mathcal{B}(] 0,+\infty[)$ e $I \subset \Sigma$.

Para todo $i \in \mathcal{I}$, podemos definir

$$
\begin{array}{rr}
\left.\left.T_{1}^{(i)}=\inf \{t>0: N(] 0, t] \times \Sigma_{i}\right)>0\right\}, & \text { se } \sum_{\sigma \in \Sigma_{i}} \nu(\sigma)>0, \\
T_{1}^{(i)}=+\infty, & \text { se } \sum_{\sigma \in \Sigma_{i}} \nu(\sigma)=0 .
\end{array}
$$

Pela hipótese (4.8) temos que $\sum_{\sigma \in \Sigma_{i}} \nu(\sigma)=\Lambda_{i}<\infty$. Portanto, se excluirmos o segundo caso, 
$T_{1}^{(i)}$ é uma variável aleatória exponencial com parâmetro $\Lambda_{i}$ e que neste instante apenas uma transformação $\sigma \in \Sigma_{i}$ ocorre, denote por $\Pi_{1}^{(i)}$ esta transformação.

Sabemos que $\Pi_{1}^{(i)}$ e $T_{1}^{(i)}$ são variáveis independente e que

$$
P\left\{\Pi_{1}^{(i)}=\sigma_{i}^{(j, k)}\right\}= \begin{cases}\frac{\lambda_{j}(k) \mathbb{I}\left\{j \in V_{\cdot \rightarrow i}^{k}\right\}}{\Lambda_{i}}, & \text { se } j \neq i \text { e } k \geq 1 \\ \frac{\lambda_{i}(k)\left(\rho_{i}\right)^{k}}{\Lambda_{i}}, & \text { se } j=i \text { e } k \geq 1 \\ \frac{\lambda_{i}(0)+\sum_{k \geq 1} \lambda_{i}(k)\left(1-\left(\rho_{i}\right)^{k}\right)}{\Lambda_{i}}, & \text { se } j=i \text { e } k=0\end{cases}
$$

Defina

$$
\tilde{C}_{1}^{(i)}=\Pi_{1}^{(i)}(\{i\})
$$

um subconjunto aleatório de $\mathcal{I}$, que representa o estado do processo $\left(C_{s}^{(i)}\right)$ após o primeiro salto. Note que a distribuição de probabilidade de $\tilde{C}_{1}^{(i)}$ é dada por

$$
P\left(\tilde{C}_{1}^{(i)}=C\right)= \begin{cases}\frac{\sum_{\sigma: \sigma(\{i\})=C} \nu(\sigma)}{\Lambda_{i}}, & \text { se } \Lambda_{i}>0 \\ 0, & \text { caso contrário. }\end{cases}
$$

Observe que $T_{1}^{(i)}$ é um tempo de parada do processo $N$ e $\tilde{C}_{1}^{(i)}$ é $\mathcal{F}_{T_{1}^{(i)}}$-mensurável. Vamos utilizar a seguinte observação para continuar a construção dos tempos de saltos $T_{n}^{(i)}$.

Seja $T$ um tempo de parada do processo $N$ e $I$ um subconjunto aleatório de $\Sigma \mathcal{F}_{T}$-mensurável, tal que $\sum_{\sigma \in I} \nu(\sigma)<\infty$.

Defina a variável aleatória $S(T, I)$ por

$$
S(T, I)= \begin{cases}\inf \{t>T: N(] T, t] \times I)>0\}, & \text { se } \mathrm{T}<\infty \text { e } \sum_{\sigma \in I} \nu(\sigma)>0, \\ +\infty, & \text { caso contrário. }\end{cases}
$$

Condicionado à $\mathcal{F}_{T}, S(T, I)$ se comporta como $T_{1}^{(i)}$, isto é,

1. se $S(T, I)$ é finito, $S(T, I)-T$ é uma variável aleatória exponencial com parâmetro $\sum_{\sigma \in I} \nu(\sigma)$ e que neste instante uma única transformação $\Pi(T, I)$ ocorre.

2. Mais ainda, $\Pi(T, I)$ é independente de $S(T, I)$ com distribuição de probabilidade dada por

$$
P(\Pi(T, I)=\sigma)= \begin{cases}\frac{\nu(\sigma)}{\sum_{\sigma^{\prime} \in I} \nu\left(\sigma^{\prime}\right)}, & \text { se } \sigma \in I, \\ 0, & \text { caso contrário. }\end{cases}
$$

Agora podemos gerar por indução

$$
\begin{array}{r}
T_{n+1}^{(i)}=S\left(T_{n}^{(i)}, \Sigma_{\tilde{C}_{n}^{(i)}}\right) \\
\tilde{C}_{n+1}^{(i)}=\Pi\left(T_{n}^{(i)}, \Sigma_{\tilde{C}_{n}^{(i)}}\right)\left(\tilde{C}_{n}^{(i)}\right) .
\end{array}
$$

Portanto, temos que as propriedades são satisfeitas e o processo de salto construído.

Prova da segunda parte: Agora vamos mostrar que, sob a hipótese adicional (4.9), o processo não explode. Para todo $n \in \mathbb{N}$ e $i \in \mathcal{I}$, temos a seguinte desigualdade

$$
\left|C_{t \wedge T_{n}^{(i)}}^{(i)}\right|=1+\sum_{\sigma} \int_{0}^{t \wedge T_{n}^{(i)}} d N^{\sigma}(s)\left(\left|\sigma\left(C_{s}^{(i)}\right)\right|-\left|C_{s}^{(i)}\right|\right)
$$


e consequentemente

$$
\mathbb{E}\left(\left|C_{t \wedge T_{n}^{(i)}}^{(i)}\right|\right)=1+\sum_{\sigma} \nu(\sigma) \mathbb{E}\left[\int_{0}^{t \wedge T_{n}^{(i)}} d(s)\left(\left|\sigma\left(C_{s}^{(i)}\right)\right|-\left|C_{s}^{(i)}\right|\right)\right] .
$$

Pela hipótese (4.9), temos que

$$
\begin{aligned}
\sum_{\sigma} \nu(\sigma)\left(\left|\sigma\left(C_{s}^{(i)}\right)\right|-\left|C_{s}^{(i)}\right|\right) & \leq \sum_{u \in C_{s}^{(i)}}\left[\sum_{\sigma} \nu(\sigma)(|\sigma(u)|-1)\right] \\
& \leq c\left|C_{s}^{(i)}\right| .
\end{aligned}
$$

De (4.16) e (4.17), temos que

$$
\begin{aligned}
\mathbb{E}\left(\left|C_{t \wedge T_{n}^{(i)}}^{(i)}\right|\right) & \leq 1+c \mathbb{E}\left[\int_{0}^{t \wedge T_{n}^{(i)}}\left|C_{s}^{(i)}\right| d s\right] \\
& \leq 1+c \mathbb{E}\left[\int_{0}^{t}\left|C_{s \wedge T_{n}^{(i)}}^{(i)}\right| d s\right] .
\end{aligned}
$$

Pela desigualdade de Gronwall, temos que

$$
\mathbb{E}\left(\left|C_{t \wedge T_{n}^{(i)}}^{(i)}\right|\right) \leq e^{c t}, \quad \text { para todo } n \in \mathbb{N} .
$$

Portanto, passando o limite em (4.18), temos que

$$
\mathbb{E}\left(\left|C_{t}^{(i)}\right|\right) \leq e^{c t}, \quad t \in \mathbb{R}_{+}
$$

Seja $T_{S T O P}^{(i)}$ o instante no qual o processo $\left(C_{s}^{(i)}\right)$ atinge o estado $\emptyset$, definido por

$$
T_{S T O P}^{(i)}=\inf \left\{s>0: C_{s}^{(i)}=\emptyset\right\} .
$$

Note que, se $T_{S T O P}^{(i)}<\infty$, temos que

$$
C_{s}^{(i)}=\emptyset
$$

para qualquer $s \geq T_{S T O P}^{(i)}$.

Também podemos definir

$$
N_{S T O P}^{(i)}=\inf \left\{n: \tilde{C}_{n}^{(i)}=\emptyset\right\}
$$

o número de passos necessário que devemos voltar no passado para determinar o conjunto dos ancestrais.

Na próxima seção vamos apresentar o pseudo-algoritmo da simulação perfeita. O pseudo-algoritmo está dividido em duas etapas. A primeira etapa envolve a simulação do processo $\left(C_{s}^{(i)}\right)$. 


\subsection{Algoritmo de simulação perfeita}

Nesta seção vamos aprensentar o pseudo-algoritmo da simulação perfeita que encontra o valor de $\xi_{0}(i)$, para qualquer $i \in \mathcal{I}$.

Divimos o algoritmo em duas etapas: (i) na primeira etapa do algoritmo, simulamos a sequência dos conjuntos dos ancestrais, (ii) na segunda etapa utilizamos a saída da primeira etapa para encontrar o valor de $\xi_{0}(i)$.

As seguintes variáveis serão utilizadas:

- $N$ é um contador assumindo valores em $\{0,1,2, \ldots\}$.

- $N_{S T O P}^{(i)}$ é uma variável assumindo valores em $\{0,1,2, \ldots\}$.

- $B$ é uma lista ordenada com elementos assumindo valores em $\mathcal{I} \times\{0,1,2, \ldots\}$.

- $C$ é uma variável assumindo valores no conjunto do subconjuntos finitos de $\mathcal{I}$.

- $V(k)$ uma variável assumindo valores no conjunto dos subconjuntos de $\mathcal{I}$.

- $p$ é um vetor com elementos assumindo valores em $\{0,1,2, \ldots\}$.

- $\xi_{0}(i)$ é um variável assumindo valores em $\{0,1,2, \ldots\}$.

Chamamos a primeira etapa do pseudo-algoritmo de Procedimento rascunho para trás. As saídas desse procedimento são $N_{S T O P}^{(i)}$, o número passos que precisamos voltar no passado para determinar o valor de $\xi_{0}(i)$, e $B$ uma lista ordenada contendo os neurônios do conjunto dos ancestrais.

As entradas do pseudo-algoritmo são a família dos grafos orientados $\left\{V_{\cdot \rightarrow j}^{k} ; j \in \mathcal{I}, k \geq 1\right\}$ e o índice do neurônio $i \in \mathcal{I}$ de interesse. 


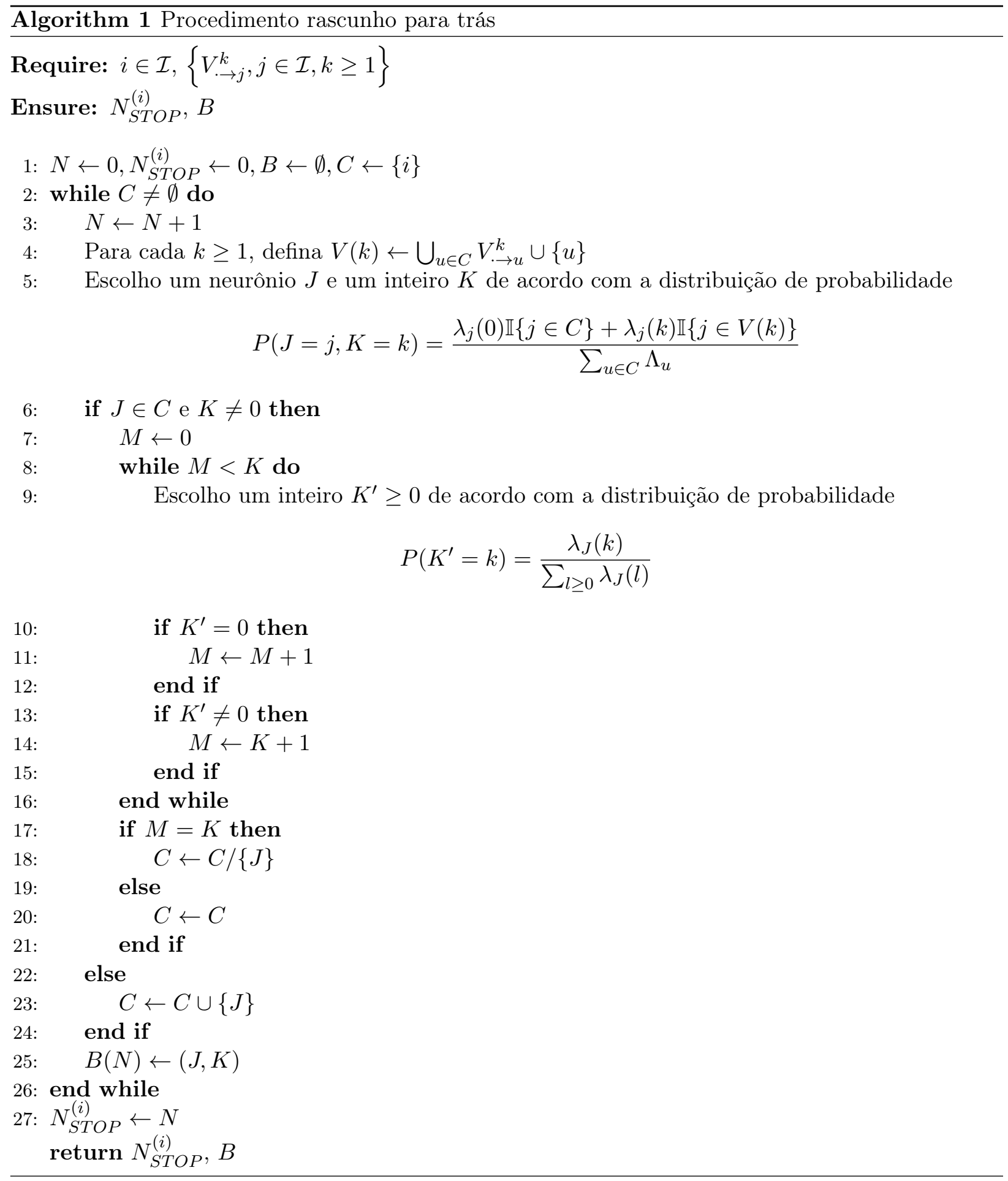

Note que o valor $N_{S T O P}^{(i)}$ que o algoritmo gera como saída é equivalente ao definido em (4.20). A saída $B$ contém as informações dos neurônios do conjunto de ancestrais. Cada linha de $B$ representa um passo do algoritmo, na primeira coordenada observamos o neurônio que foi escolhido e na segunda o valor $k$ da transformação.

Observe que o neurônio da linha $N_{S T O P}^{(i)}$ da lista $B$ é o único neurônio presente no conjunto dos ancestrais. No passo $N_{S T O P}^{(i)}$ do algoritmo, sabemos que o valor do potencial de membrana deste neurônio vai para 0. Esta é a ideia da segunda parte do algoritmo, começando da última até a primeira linha de $B$, conseguimos definir se cada transformação que ocorreu, na primeira etapa do pseudo-algoritmo, afeta efetivamente o neurônio $i$ no instante $t=0$.

A cada passo que subimos a lista $B$, podemos encontrar neurônios que não foram observados anteriormente. Quando isto acontece, sabemos que o valor do potencial de membrana deste neurônio 
vai para 0 e que o potencial de ação gerado por este neurônio aumenta em uma unidade o potencial de membrana dos neurônios que são influenciados por ele, inclusive o neurônio $i$.

A segunda etapa, chamada de Procedimento atribuição para frente, utiliza $B$ e $N_{S T O P}^{(i)}$ como entradas e gera como saída o valor de $\xi_{0}(i)$

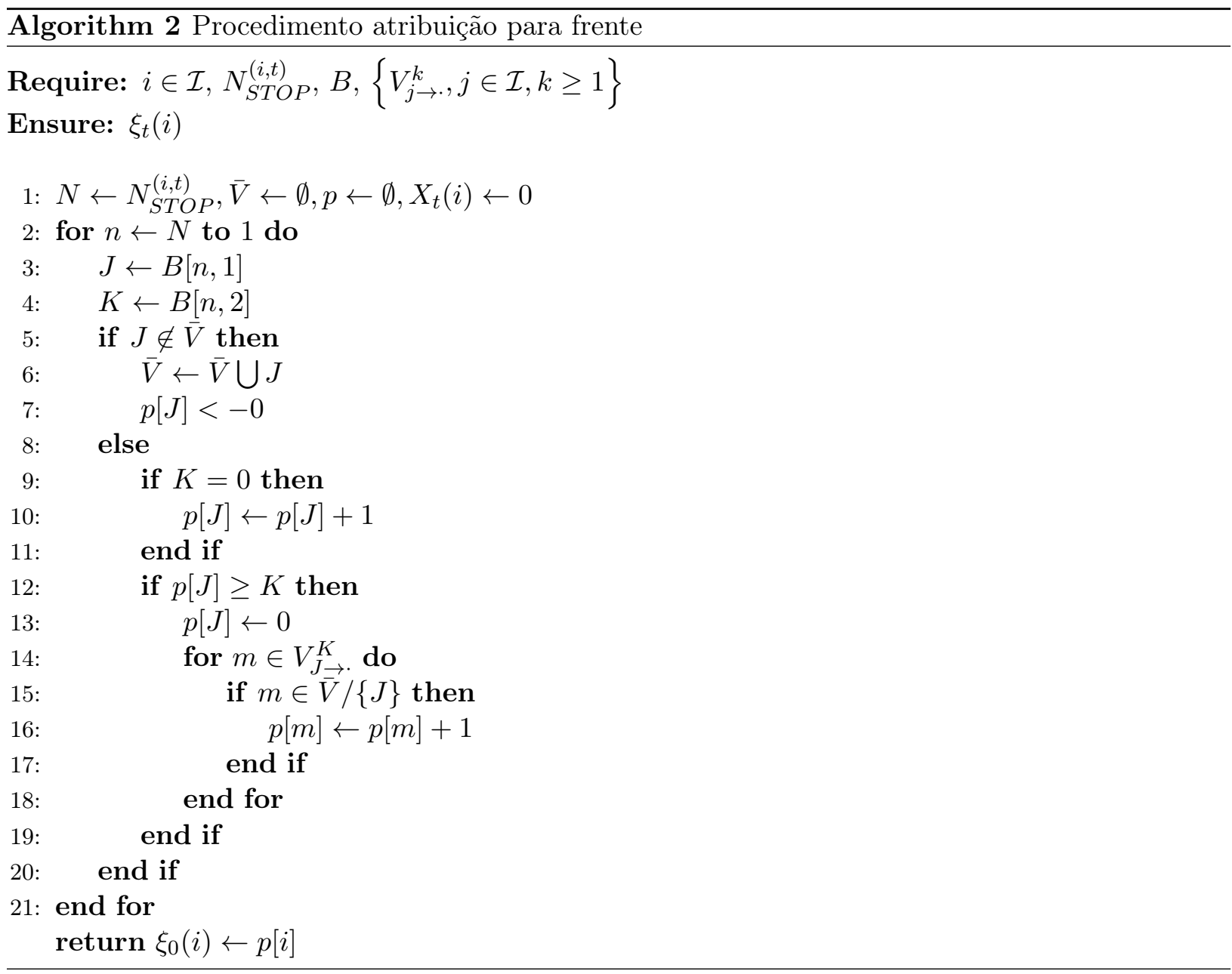

Dizemos que o algoritmo de simulação perfeita é bem sucedido se $N_{S T O P}^{(i)}$ é finito quase certamente. Este é o conteúdo do próximo teorema.

Teorema 4.3.1. Sob a condição

$$
\sup _{i \in \mathcal{I}} \sum_{\sigma \in \Sigma_{i}} \frac{\nu(\sigma)|\sigma(\{i\})|}{\Lambda_{i}}=\alpha \leq 1
$$

temos que para qualquer $i \in \mathcal{I}$,

$$
\mathbb{P}\left\{N_{S T O P}^{(i)}>n\right\} \leq \alpha^{n}
$$

$e$

$$
\mathbb{E}\left(N_{S T O P}^{(i)}\right) \leq \frac{1}{1-\alpha}
$$

Para todo $i \in \mathcal{I}$, temos que

$$
\sum_{\sigma \in \Sigma_{i}} \frac{\nu(\sigma)|\sigma(\{i\})|}{\Lambda_{i}}=\frac{\lambda_{i}(0)+\sum_{k \geq 1} \lambda_{i}(k)\left(1-\left(\rho_{i}\right)^{k}\right)+2 \sum_{k \geq 1} \sum_{j \in V_{\cdot \rightarrow i}^{k}} \lambda_{j}(k)}{\Lambda_{i}} .
$$




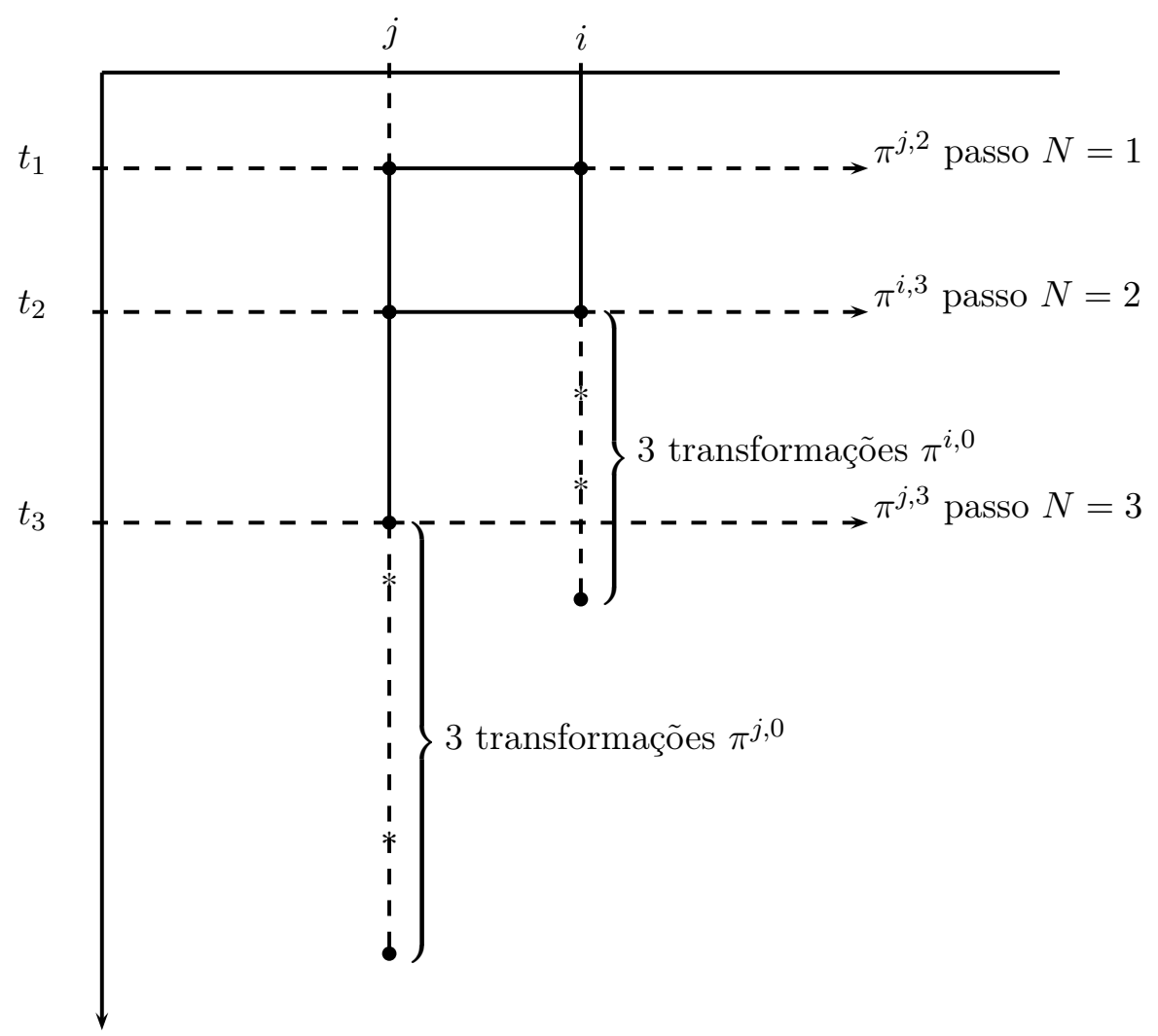

Figura 4.1: Exemplo de simulação perfeita

Para que a condição (4.21) seja satisfeita precisamos que, para todo $i \in \mathcal{I}$,

$$
\lambda_{i}(0)+\sum_{k \geq 1} \lambda_{i}(k)\left(1-\left(\rho_{i}\right)^{k}\right)+2 \sum_{k \geq 1} \sum_{j \in V_{\cdot \rightarrow i}^{k}} \lambda_{j}(k) \leq \Lambda_{i}
$$

Portanto, a condição (4.21) é satisfeita se

$$
\sum_{k \geq 1} \sum_{j \in V_{\cdot \rightarrow i}^{k}} \lambda_{j}(k) \leq \sum_{k \geq 1} \lambda_{i}(k)\left(\rho_{i}\right)^{k}
$$

para todo $i \in \mathcal{I}$. Note que esta condição é igual a condição (3.5) do Teorema 3.2.1. Vamos apresentar a prova do Teorema 4.3.1 no Capítulo 6 .

Na Figura 4.1 ilustramos algumas etapas do Algoritmo 1. Apresentamos um caso específico de possíveis eventos, começamos o algoritmo obsevando apenas o que acontece no passado do neurônio $i$. No primeiro passo do algoritmo, $N=1$, sorteamos $\left(J_{1}^{(i)}, K_{1}^{(i)}\right)=(j, 2)$ o que é equivalente a sortear a transformação $\pi^{j, 2}$. Neste instante, adicionamos $j$ ao conjunto $C$ e começamos a observar também o passado do neurônio $j$.

Em $N=2$, é sorteado o par $\left(J_{2}^{(i)}, K_{2}^{(i)}\right)=(i, 3)$ e em seguida verificamos que ocorreram pelo menos três marcas $\pi^{i, 0}$, logo podemos retirar o neurônio $i$ do conjunto $C$. No passo $N=3$, observamos o mesmo evento que no passo $N=2$ para o neurônio $j$, portanto $C=\emptyset$ e o algoritmo chega ao final e retorna os valores

$$
N_{S T O P}^{(i)}=3 \quad \text { e } \quad B=\left[\begin{array}{cc}
j & 2 \\
i & 3 \\
j & 3
\end{array}\right] .
$$

Agora podemos definir, usando o Algoritmo 2, o valor de $\xi_{0}(i)$. Com essas saídas, sabemos que em $N=3$ o valor de $\xi_{t_{3}}(j)=0$, então em $N=1$ a transformação $\pi^{j, 2}$ não altera o potencial de 
membrana do neurônio $i$, pois $\xi_{t_{1}-}(j)<2$. Como no passo $N=2, \xi_{t_{2}}=0$ e a transformação no passo $N=1$ não alterou o valor do potencial de membrana do neurônio $i$, temos que $\xi_{0}(i)=0$. Concluindo o algoritmo de simulação perfeita. 


\section{Capítulo 5}

\section{Um algoritmo de simulação perfeita para o processo acoplado $\left(\xi_{t}, \xi_{t}^{[F]}\right)_{t \in \mathbb{R}}$}

No processo apresentado no Capítulo 3, consideramos um sistema com infinitas componentes. Podemos nos perguntar o que acontece com o processo quando consideramos apenas um subconjunto finito de neurônios, em vez de considerarmos todos os neurônios do sistema.

Neste capítulo, nos dedicamos a responder essa pergunta. Para isso, vamos definir primeiramente o processo $\left(\xi_{t}^{[F]}\right)_{t \in \mathbb{R}}$ limitado ao conjunto finito $F$, assumindo valores em $\{0,1, \ldots\}^{F}$. Depois vamos introduzir um algoritmo de simulação perfeita para o acoplamento entre os processos $\left(\xi_{t}\right)$ e $\left(\xi_{t}^{[F]}\right)$, isto é, um algoritmo que constrói simultaneamente os dois processos através do mesmo dispositivo aleatório.

Como consequência obtemos um limitante superior para a probabilidade da discrepância entre $\xi_{t}(i)$ e $\xi_{t}^{[F]}(i)$, para todo $F \subset \mathcal{I}$ finito, $i \in \mathcal{I}$ e $t \in \mathbb{R}$, quando utilizamos o algoritmo de simulação perfeita para gerar uma amostra do processo acoplado $\left(\xi_{t}, \xi_{t}^{[F]}\right)$.

\subsection{Definições e resultado}

Vamos definir um sistema markoviano de partículas em $F \subset \mathcal{I}$ finito com espaço de estados $\mathbb{N}$. Denote por $\mathcal{S}^{[F]}=\mathbb{N}^{F}$ o espaço das configurações do sistema. Uma configuração de $\mathcal{S}^{[F]}$ será denotado por $\xi^{[F]}$.

A configuração do sistema no instante $t \in \mathbb{R}$ é denotada por

$$
\xi_{t}^{[F]}=\left(\xi_{t}^{[F]}(i), i \in F\right),
$$

onde $\xi_{t}^{[F]}(i) \in \mathbb{N}$, para $i \in F$.

Seja $\left\{\pi^{i, k,[F]} ; i \in \mathcal{I}, k \geq 0\right\}$ uma família de transformações restrita ao conjunto $F$. Para $k \geq 1$,

$$
\pi^{i, k,[F]}\left(\xi^{[F]}\right)(u)= \begin{cases}\xi^{[F]}(u), & \text { se } \xi^{[F]}(i)<k \\ \xi^{[F]}(u), & \text { se } \xi^{[F]}(i) \geq k, u \neq i \text { e } u \notin V_{i \rightarrow .}^{k} .(F) \\ \xi^{[F]}(u)+1, & \text { se } \xi^{[F]}(i) \geq k, u \neq i \text { e } u \in V_{i \rightarrow .}^{k} .(F) \\ 0, & \text { se } \xi^{[F]}(i) \geq k \text { e } u=i .\end{cases}
$$

Para $k=0$,

$$
\pi^{i, 0,[F]}\left(\xi^{[F]}\right)(u)= \begin{cases}\xi^{[F]}(u), & \text { se } u \neq i \\ 0, & \text { se } u=i\end{cases}
$$


onde, para $i \in \mathcal{I}$ e $k \geq 1$,

$$
V_{\cdot \rightarrow i}^{k}(F)=V_{\cdot \rightarrow i}^{k} \cap F,
$$

é o conjunto dos neurônios que influenciam o neurônio $i$ e que pertencem ao conjunto $F$.

As transformções $\left\{\pi^{i, k,[F]}\right\}$ são as restrições naturais ao conjunto das configurações em $\mathcal{S}^{[F]}$ das transformações $\left\{\pi^{i, k}\right\}$ definidas no Capítulo 3.

O gerador infinitesimal do processo $\left(\xi_{t}^{[F]}\right)_{t \in \mathbb{R}}$ é dado por

$$
\mathcal{L}^{[F]} f\left(\xi^{[F]}\right)=\sum_{i \in F} \sum_{k \geq 0} \lambda_{i}(k)\left[f\left(\pi^{i, k}\left(\xi^{[F]}\right)\right)-f\left(\xi^{[F]}\right)\right]
$$

onde $f: \mathcal{S}^{[F]} \rightarrow \mathbb{R}$ é qualquer função cilíndrica limitada. As funções $\left\{\lambda_{i}(k) ; i \in F, k \geq 0\right\}$ são as mesmas definidas no Capítulo 3.

Podemos definir da mesma maneira a evolução do processo rascunho para trás $\left(C_{s}^{(i),[F]}\right)_{s \geq 0}$, associado ao processo limitado ao conjunto $F$.

Seja $\Sigma(F)=\left\{\sigma_{i}^{(j, k, F)}: i, j \in F, k \geq 0\right\}$ uma família de transformações em $\mathcal{P}(F)$, o conjunto dos subconjuntos de $F$, definidas da seguinte maneira. Para qualquer conjunto unitário $\{u\} \in \mathcal{P}(F)$, para $j \neq i$ e $k \geq 1$,

$$
\sigma_{i}^{(j, k, F)}(\{u\})= \begin{cases}\{i, j\}, & \text { se } u=i \text { e } j \in V_{\cdot \rightarrow i}^{k}(F) \\ \{u\}, & \text { caso contrário. }\end{cases}
$$

Para $j=i$ e $k \geq 1$,

$$
\sigma_{i}^{(i, k, F)}(\{u\})= \begin{cases}\emptyset, & \text { se } u=i \\ \{u\}, & \text { caso contrário. }\end{cases}
$$

Para $j=i$ e $k=0$,

$$
\sigma_{i}^{(i, 0)}(\{u\})=\{u\} \text { para todo } u .
$$

Para qualquer conjunto $C \in \mathcal{P}(F)$, defina de forma semelhante

$$
\sigma_{i}^{(j, k, F)}(C)=\bigcup_{u \in C} \sigma_{i}^{(j, k, F)}(\{u\})
$$

A família de transformações $\Sigma(F)$ é uma restrição natural ao conjunto $\mathcal{P}(F)$ da família de transformações $\Sigma$ definida no Capítulo 4.

Agora podemos definir o processo $\left(C_{s}^{(i),[F]}\right)$ assumindo valores em $\mathcal{P}(F)$, com condição inicial $C_{0}^{(i),[F]}=\{i\}$, pelo gerador infinitesimal dado por

$$
\mathcal{G}^{[F]} f(C)=\sum_{\sigma \in \Sigma(F)} \nu(\sigma)[f(\sigma(C))-f(C)]
$$

onde $f: \mathcal{P}(F) \rightarrow \mathbb{R}$ é qualquer função cilíndrica limitada e $C \in \mathcal{P}(F)$.

Note que a função $\nu(\cdot)$ é a mesma definida no Capítulo 4 . Denote por $\mathcal{P}_{f}(\mathcal{I})$ o conjunto dos subconjuntos finitos de $\mathcal{I}$.

A próxima proposição resume as propriedades do processo rascunho para trás, associado ao processo limitado ao conjunto $F$.

Proposição 5.1.1. Para $F \in \mathcal{P}_{f}(\mathcal{I})$, suponha que

$$
\sum_{\sigma \in \Sigma_{i}(F)} \nu(\sigma)<\infty
$$


para todo $i \in F$, onde $\Sigma_{i}(F)=\left\{\sigma_{i}^{(j, k, F)}: k \geq 0, j \in V_{\cdot \rightarrow i}^{k}(F) \cup\{i\}\right\}$. Então, existe um espaço de probabilidade $(\Omega, \mathcal{B}, P)$ sobre o qual podemos construir, para todo $i \in F$, uma versão do processo markoviano de salto $\left(C_{s}^{(i),[F]}, s \in \mathbb{R}_{+}\right)$em $\mathcal{P}(F)$, com condição inicial $\{i\}$ e com gerador infinitesimal $\mathcal{G}^{[F]}$ definido em (5.8).

Demonstração. A construção do processo $\left(C_{s}^{(i),[F]}\right)$ é análoga a construção do processo $\left(C_{s}^{(i)}\right)$ apresentado na demonstração da Proposição 4.2.1, a única diferença é que a construção do processo é restrita ao conjunto $F$.

Para todo $F$ subconjunto finito de $\mathcal{I}$ e $i \in \mathcal{I}$, temos que $\left|C_{s}^{(i),[F]}\right| \leq|F|$ para qualquer $s \in \mathbb{R}_{+}$. Portanto, não precisamos mostrar que o processo $\left(C_{s}^{(i),[F]}\right)$ não explode.

Denote por $\left(\tilde{C}_{n}^{(i),[F]}\right)$ a cadeia de Markov em $\mathcal{P}(F)$ associada ao processo $\left(C_{s}^{(i),[F]}\right)$. Defina por

$$
N_{S T O P}^{(i),[F]}=\inf \left\{n: \tilde{C}_{n}^{(i),[F]}=\emptyset\right\}
$$

o número de passos necessários que devemos voltar no passado para determinar o conjunto dos ancetrais do processo $\left(C_{s}^{(i),[F]}\right)$.

No próximo teorema, apresentamos o resultado sobre o limitante superior da probabilidade da discrepância entre os dois processos.

Teorema 5.1.1. Sob as hipóteses do Teorema 3.2.1. Para todo $F \in \mathcal{P}_{f}(\mathcal{I})$ e $i \in F$, existe um algoritmo de acoplamento por simulação perfeita entre os processos $(\xi(i))$ e $\left(\xi^{[F]}(i)\right)$, satisfazendo a seguinte desigualdade

$$
P\left\{\xi(i) \neq \xi^{[F]}(i)\right\} \leq \frac{\delta(F)}{1-\alpha}
$$

onde $\delta(F) \rightarrow 0$ quando $F \rightarrow \mathcal{I}$, definido em (6.26)

O Teorema 5.1.1, diz que a probabilidade do algoritmo de simulação perfeita para o processo acoplado gerar saídas tais que $\xi_{t}(i) \neq \xi_{t}^{[F]}(i)$ é limitada superiormente por um fator que depende do conjunto $F$ e que quando $F \rightarrow \mathcal{I}$ a probabilidade dos valores amostrados serem diferentes vai para zero.

Para provar o Teorema 5.1.1, precisamos construir um algoritmo de simulação perfeita para o processo acoplado e garantir que o algoritmo é bem sucedido. Além disso, precisamos mostrar que o acoplamento construído pelo algoritmo satisfaz a desigualdade em (5.11).

Na próxima seção vamos apresentar o algoritmo de simulação perfeita para o acoplamento entre o processo limitado ao conjunto $F$ e o processo que considera todo o sistema. A ideia é construir simultaneamente os processos $\left(C_{s}^{(i)}\right)$ e $\left(C_{s}^{(i),[F]}\right)$.

\subsection{Simulação perfeita para o aclopamento dos processos $\left(\xi_{t}, \xi_{t}^{[F]}\right)_{t \in \mathbb{R}}$}

Nesta seção vamos apresentar o pseudo-algoritmo de simulação perfeita para o acoplamento entre os processos $\left(\xi_{t}(i)\right)$ e $\left(\xi_{t}^{[F]}(i)\right)$, para todo $F \subset \mathcal{I}$ finito e $i \in F$. Sem perda de generalidade, vamos assumir novamente que $t=0$.

Queremos construir de forma simultânea os conjuntos dos ancestrais para os dois processos. Ou seja, vamos construir um acoplamento entre os processos $\left(C_{s}^{(i)}\right)$ e $\left(C_{s}^{(i),[F]}\right)$.

Proposição 5.2.1. Para todo $F \subset \mathcal{I}$ finito $e i \in F$, existe um acoplamento entre os processos $\left(C_{s}^{(i)}\right)$ e $\left(C_{s}^{(i),[F]}\right)$ tal que

$$
C_{s}^{(i),[F]} \subseteq C_{s}^{(i)}, \text { para todo } s \in \mathbb{R}_{+}
$$


Demonstração. Seja $\left(T_{n}^{(i)}\right)_{n \geq 0}$ a sequência dos tempos de saltos definidos no Capítulo 4. Para todo $F \in \mathcal{P}_{f}(F)$ e $i \in F$, vamos construir o acoplamento entre os processos $\left(C_{s}^{(i)}\right)$ e $\left(C_{s}^{(i),[F]}\right)$ da seguinte maneira:

1. Para $t \in\left[0, T_{1}^{(i)}[\right.$, defina

$$
C_{t}^{(i)}=C_{t}^{(i),[F]}=\{i\}
$$

2. Para $n \geq 1$ :

(a) Dado $C_{T_{n-1}^{(i)}}^{(i)}$, sorteamos uma transformação $\Pi_{n}$ com distribuição de probabilidade dada por

$$
P\left(\Pi_{n}=\sigma\right)= \begin{cases}\frac{\nu(\sigma)}{\sum_{\sigma^{\prime} \in \Sigma_{n}} \nu\left(\sigma^{\prime}\right)}, & \text { se } \sigma \in \Sigma_{n}, \\ 0, & \text { caso contrário, }\end{cases}
$$

onde $\Sigma_{n}=\left\{\sigma_{u}^{(j, k)} \in \Sigma: u \in C_{T_{n-1}^{(i)}}^{(i)}, k \geq 0, j \in V_{\cdot \rightarrow u}^{k} \cup\{u\}\right\}$.

(b) Defina para $T_{n}^{(i)} \leq t<T_{n+1}^{(i)}$,

$$
C_{t}^{(i)}=\Pi_{n}\left(C_{T_{n-1}^{(i)}}^{(i)}\right)
$$

e

$$
C_{t}^{(i),[F]}= \begin{cases}\Pi_{n}\left(C_{T_{n-1}^{(i),[F]}}^{(i)}\right), & \text { se } \Pi_{n} \in \Sigma_{n}(F), \\ C_{T_{n-1}^{(i),[}}^{(i)}, & \text { caso contrário, }\end{cases}
$$

onde $\Sigma_{n}(F)=\left\{\sigma_{u}^{(j, k)} \in \Sigma: u \in C_{T_{n-1}^{(i)}}^{(i)} \cap F, k \geq 0, j \in V_{\cdot \rightarrow u}^{k}(F) \cup\{u\}\right\}$.

Portanto, pela construção do acoplamento, temos que

$$
C_{s}^{(i),[F]} \subseteq C_{s}^{(i)} \text { para qualquer } s \in \mathbb{R}_{+} .
$$

Portanto, quando construímos os dois processos simultaneamente, utilizando as mesmas variáveis uniformes para os sorteios dos neurônios e dos valores $k$ das transformações, temos que se o neurônio sorteado pertence ao conjunto $F$, incluímos, retiramos ou mantemos o neurônio nos dois processos. Caso contrário, o sorteio apenas alterá o processo que considera todo o sistema.

Como consequência da proposição 5.2.1, temos o seguinte corolário.

Corolário 5.2.1. Para $F \in \mathcal{P}_{f}(\mathcal{I})$ e $i \in F$, temos que

$$
N_{S T O P}^{(i),[F]} \leq N_{S T O P}^{(i)}
$$

Demonstração. Segue da construção do acoplamento definido na demonstração da Proposição 5.2.1.

Agora podemos apresentar o pseudo-algoritmo de simulação perfeita para o acoplamento entre os processos $\left(\xi_{t}\right)$ e $\left(\xi_{t}^{[F]}\right)$, para qualquer $F \in \mathcal{P}_{f}(\mathcal{I})$. 


\subsubsection{Algoritmo}

Vamos construir um pseudo-algoritmo de simulação perfeita que gera como saída o valores de $\xi_{0}(i)$ e $\xi_{0}^{[F]}(i)$ quando acoplamos os dois processos, para qualquer $F \in \mathcal{P}_{f}(\mathcal{I})$ e $i \in F$.

Como no pseudo-algoritmo apresentado anteriormente, dividimos o procedimento em duas etapas. Na primeira etapa, construímos simultaneamente os conjuntos dos ancestrais $\left(C_{s}^{(i)}\right)$ e $\left(C_{s}^{(i),[F]}\right)$.

A segunda etapa é idêntica ao Algoritmo 2 apresentado no Capítulo 4. Nesta etapa utilizamos as saídas $B$ e $N_{S T O P}^{(i)}$ para gerar o valor $\xi_{0}(i)$ e as saídas $B^{[F]}$ e $N_{S T O P}^{(i),[F]}$ para gerar o valor $\xi_{0}^{[F]}(i)$.

A construção do pseudo-algoritmo é semelhante ao apresentado no Capítulo 4, apenas precisamos incluir alguns passos que garante o acoplamento entre os dois processos ao procedimento.

As seguintes variáveis serão utilizadas:

- $N, N^{[F]}$ são contadores assumindo em $\{0,1,2, \ldots\}$.

- $N_{S T O P}^{(i)}, N_{S T O P}^{(i),[F]}$ são variáveis assumindo valores em $\{0,1,2, \ldots\}$.

- $B, B^{[F]}$ são listas ordenadas com elementos assumindo valores em $\mathcal{I} \times\{0,1,2, \ldots\}$.

- $C, C^{[F]}$ são variáveis assumindo valores no conjunto dos sunconjuntos de $\mathcal{I}$.

- $V(k)$ é uma variável assumindo valores no conjunto dos sunconjuntos de $\mathcal{I}$.

- $p$ é um vetor com elementos assumindo valores em $\{0,1,2, \ldots\}$.

- $\xi(i), \xi(i)^{[F]}$ são variáveis assumindo valores em $\{0,1,2, \ldots\}$.

As entradas do algoritmo é a família de grafos $\left\{V_{\cdot \rightarrow j}^{k}, j \in \mathcal{I}, k \geq 1\right\}$, o conjunto $F \in \mathcal{P}_{f}(\mathcal{I})$ e o índice do neurônio $i \in F$ de interesse. As saídas desse procedimento são $N_{S T O P}^{(i)}, N_{S T O P}^{(i),[F]}, B$ e $B^{[F]}$. 


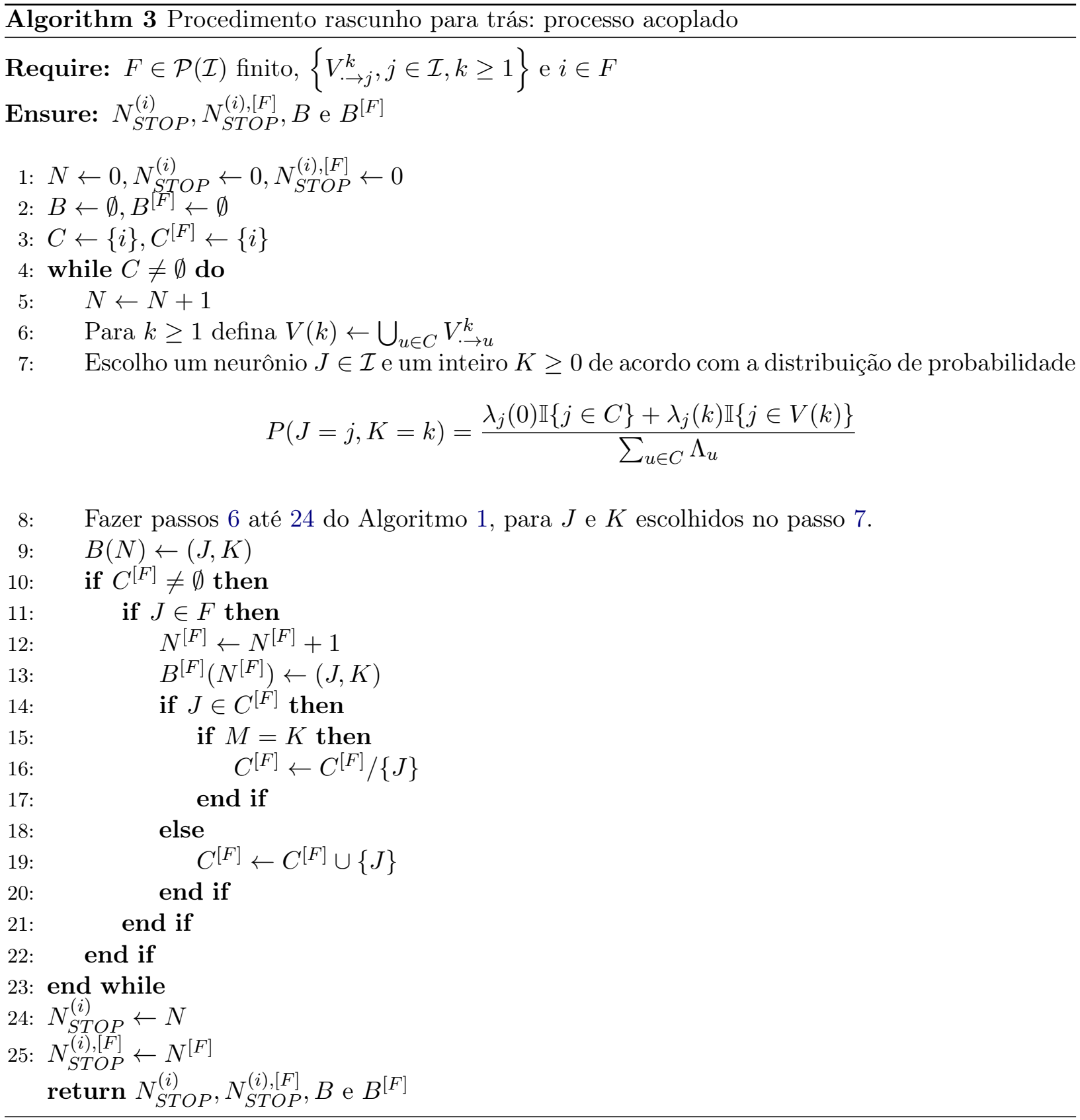

Usamos o Algoritmo 2 para determinar o valor de $\xi_{0}(i)$ e $\xi_{0}^{[F]}(i)$. Se durante a construção dos conjuntos dos ancestrais nenhum neurônio $j$, tal que $j \notin F$, foi sorteado, temos que $B=B^{[F]}$ e consequentemente $\xi_{0}(i)=\xi_{0}^{[F]}(i)$.

Caso contrário, temos que $B \neq B^{[F]}$. Neste caso, utilizamos o Algoritmo 2 para determinar o valor $\xi_{0}(i)$ com as entradas $N_{S T O P}^{(i)}$ e $B$. E independentemente, determinamos o valor de $\xi_{0}^{[F]}(i)$ com as entradas $N_{S T O P}^{(i),[F]}$ e $B^{[F]}$.

Agora precisamos mostrar que o algoritmo construído é bem sucedido. Ou seja, precisamos mostrar que o número de passos do algoritmo é finito quase certamente. Pelo Corolário 5.2.1, temos que

$$
N_{S T O P}^{(i)} \geq N_{S T O P}^{(i),[F]}
$$

Portanto, precisamos mostrar apenas que $N_{S T O P}^{(i)}$ é finito. Observe que $N_{S T O P}^{(i)}$ é equivalente ao do Algoritmo 1 e pelo Teorema 3.2.1 temos que $N_{S T O P}^{(i)}$ é finito quase certamente.

Para concluir a prova do Teorema 5.1.1, falta apenas mostrar que a desigualdade em (5.11) é satisfeita. Esta será apresentada no Capítulo 6. 


\section{Capítulo 6}

\section{Prova dos resultados}

Este capítulo é dedicada a prova dos resultados apresentados nesta tese. Na primeira seção, vamos apresentar a prova do Teorema 4.3.1, o teorema fornece um limitante superior para a probabilidade do número de passos do Algoritmo 1 ser maior que um dado $n \in \mathbb{Z}_{+}$. Além disso, encontramos um limitante superior para o valor esperado do número de passos do algoritmo.

A prova da existência e unicidade do processo que introduzimos nesta tese é baseada na possibilidade da realização de um procedimento de simulação perfeita que amostra o valor de $\xi_{t}(i)$, para todo $i \in \mathcal{I}$.

Apresentamos a construção do procedimento de simulação perfeita no Capítulo 4. Aqui vamos mostrar que o Algoritmo 1 é bem sucedido, isto é, precisamos mostrar que o número de passos do algoritmo $N_{S T O P}^{(i)}$ é finito quase certamente, para todo $i \in \mathcal{I}$. A prova do Teorema 3.2.1, utiliza o resultado apresentando no Teorema 4.3.1.

Finalizamos este capítulo com a prova do Teorema 5.1.1, mostramos que o acoplamento construído pelo Algoritmo 3 satisfaz a desigualdade em (5.11).

\subsection{Prova do Teorema 4.3.1}

\subsubsection{Prova da primeira parte}

Vamos começar provando a desigualdade em (4.22). Seja

$$
Z_{n}^{(i)}=\left|\tilde{C}_{n}^{(i)}\right|
$$

o cardinal do conjunto $\tilde{C}_{n}^{(i)}$ depois de $n$ passos do Algoritmo 1.

Para todo $i \in \mathcal{I}$, temos que

$$
\begin{aligned}
P\left\{N_{\text {STOP }}^{(i)}>n\right\} & =P\left\{\left|\tilde{C}_{n}^{(i)}\right| \geq 1\right\} \\
& \leq \sum_{m=1}^{\infty} P\left\{\left|\tilde{C}_{n}^{(i)}\right| \geq m\right\} \\
& =\mathbb{E}\left[\left|\tilde{C}_{n}^{(i)}\right|\right] .
\end{aligned}
$$

Dado $\tilde{C}_{n}^{(i)}=C, C \in \mathcal{P}_{f}(\mathcal{I})$, o valor esperado de $Z_{n+1}$ é dado por

$$
\mathbb{E}\left(Z_{n+1} \mid \tilde{C}_{n}^{(i)}=C\right)=\sum_{u \in C} \sum_{\sigma \in \Sigma_{u}} \frac{\nu(\sigma)}{\sum_{\sigma \in \Sigma_{u}} \nu(\sigma)}|\sigma(u)| .
$$


Pela condição (4.21), temos que

$$
\mathbb{E}\left(Z_{n+1}\right) \leq \sum_{u \in C} \alpha=|C| \alpha .
$$

Portanto,

$$
\begin{aligned}
\mathbb{E}\left(Z_{n+1}\right) & =\mathbb{E}\left[\mathbb{E}\left(Z_{n+1} \mid \tilde{C}_{n}^{(i)}\right)\right] \\
& \leq \alpha \mathbb{E}\left(Z_{n}\right) .
\end{aligned}
$$

Recursivamente, temos que

$$
\mathbb{E}\left(Z_{n}\right) \leq \alpha^{n}
$$

Substituindo (6.2) em (6.1), temos que

$$
P\left\{N_{S T O P}^{(i)}>n\right\} \leq \alpha^{n}
$$

Concluindo a primeira parte da demonstração.

\subsubsection{Prova da segunda parte}

Seja $\left\{Y_{n}^{(i)} ; n \in \mathbb{N}, i \in \mathcal{I}\right\}$ uma família de variáveis aleatórias indenpendentes com distribuição de probabilidade que depende de $i$, independentes do processo $\left(\tilde{C}_{n}^{(i)}\right)$, assumindo valores em $\{-1,1\}$.

Para $i \in \mathcal{I}$, a distribuição de probabilidade de $Y_{n}^{(i)}$ é dada por

$$
\begin{aligned}
P\left\{Y_{n}^{(i)}=-1\right\} & =\frac{\sum_{k \geq 1} \lambda_{i}(k)\left(\rho_{i}\right)^{k}}{\Lambda_{i}}, \\
P\left\{Y_{n}^{(i)}=0\right\} & =\frac{\lambda_{i}(0)+\sum_{k \geq 1} \lambda_{i}(k)\left(1-\left(\rho_{i}\right)^{k}\right)}{\Lambda_{i}}, \\
P\left\{Y_{n}^{(i)}=1\right\} & =\frac{\sum_{k \geq 1} \sum_{j \in V_{\rightarrow i}^{k}} \lambda_{j}(k)}{\Lambda_{i}} .
\end{aligned}
$$

O valor esperado de $Y_{n}^{(i)}$ é dado por

$$
\begin{aligned}
\mathbb{E}\left[Y_{n}^{(i)}\right] & =\frac{1}{\Lambda_{i}}\left(-\sum_{k \geq 1} \lambda_{i}(k)\left(\rho_{i}\right)^{k}+\sum_{k \geq 1} \sum_{j \in V_{: \rightarrow i}^{k}} \lambda_{j}(k)\right) \\
& =\frac{1}{\Lambda_{i}}\left(\lambda_{i}(0)+\sum_{k \geq 1} \lambda_{i}(k)\left(1-\left(\rho_{i}\right)^{k}\right)+2 \sum_{k \geq 1} \sum_{j \in V_{: \rightarrow i}^{k}} \lambda_{j}(k)\right)-1 .
\end{aligned}
$$

Pela condição (4.21), temos que

$$
\sup _{i \in \mathcal{I}} \mathbb{E}\left[Y_{1}^{(i)}\right]=\alpha-1 \leq 0,
$$

onde $\alpha$ é definido em (4.21).

Para qualquer $i \in \mathcal{I}$, denote por $J_{n}^{(i)}$ o índice do neurônio cujo o relógio Poisson tocou no $n$-ésimo salto do processo $\left(C_{s}^{(i)}\right)$. Lembrando que $J_{n}^{(i)}$ são condicionalmente independentes dado a 
sequência $\left(\tilde{C}_{n}^{(i)}\right)_{n}$ tal que

$$
P\left(J_{n}^{(i)}=j \mid \tilde{C}_{n-1}^{(i)}\right)= \begin{cases}\frac{\Lambda_{j}}{\sum_{u \in \tilde{C}_{n-1}^{(i)}} \Lambda_{u}}, & \text { se } j \in \tilde{C}_{n-1}^{(i)} \\ 0, & \text { caso contrário. }\end{cases}
$$

Defina $\left(S_{n}\right)_{n \geq 0}$ um processo assumindo valores em $\mathbb{Z}$, com condição inicial $S_{0}=0$, e para $n \geq 1$

$$
S_{n}=\sum_{m=1}^{n} Y_{m}^{J_{m}^{(i)}}
$$

Note que $M_{n}=S_{n}+(1-\alpha) n$ é um super martingal,

$$
\begin{aligned}
\mathbb{E}\left(S_{n}+(1-\alpha) n \mid S_{n-1}\right) & =\mathbb{E}\left(Y_{n}^{J_{n}^{(i)}}\right)+S_{n-1}+(1-\alpha) n \\
& \leq \alpha-1+S_{n-1}+(1-\alpha) n \\
& =S_{n-1}+(1-\alpha)(n-1)
\end{aligned}
$$

Por construção, temos que

$$
\left|\tilde{C}_{n}^{(i)}\right| \leq 1+S_{n}
$$

quando $n \leq V_{S T O P}$, onde $V_{S T O P}=\min \left\{n: S_{n}=-1\right\}$. Então

$$
N_{S T O P}^{(i)} \leq V_{S T O P}
$$

Como $M_{n}$ é um super martingal e $V_{S T O P}$ é um tempo de parada temos que $M_{n \wedge V_{S T O P}}$ é um super martingal. Para $N$ fixado, temos que

$$
\begin{aligned}
\mathbb{E}\left[S_{V_{S T O P} \wedge N}+(1-\alpha)\left(V_{S T O P} \wedge N\right)\right] & =\mathbb{E}\left[S_{V_{S T O P} \wedge N}\right]+(1-\alpha) \mathbb{E}\left[V_{S T O P} \wedge N\right] \\
& \leq \mathbb{E}\left[M_{0}\right]=0
\end{aligned}
$$

Note que

$$
\mathbb{E}\left(S_{V_{S T O P} \wedge N}\right)=-1 P\left\{V_{S T O P} \leq N\right\}+\mathbb{E}\left(S_{V_{S T O P} \wedge N} ; V_{S T O P}>N\right) .
$$

Em $\left\{V_{S T O P}>N\right\}, S_{N} \geq 0$, consequentemente temos que $\mathbb{E}\left(S_{V_{S T O P} \wedge N}\right) \geq-P\left\{V_{S T O P} \leq N\right\}$. Então, concluímos que

$$
\mathbb{E}\left(V_{S T O P} \wedge N\right) \leq \frac{P\left\{V_{S T O P} \leq N\right\}}{1-\alpha}
$$

Agora, quando $N \rightarrow \infty$, temos que

$$
\mathbb{E}\left(V_{S T O P}\right) \leq \frac{1}{1-\alpha},
$$

e portanto

$$
\mathbb{E}\left(N_{S T O P}^{(i)}\right) \leq \frac{1}{1-\alpha} .
$$




\subsection{Prova do Teorema 3.2.1}

Queremos mostrar a existência e unicidade do processo $\left(\xi_{t}\right)_{t \in \mathbb{R}}$. Para isso, construimos um procedimento de simulação perfeita que gera como saída o valor da configuração $\xi_{0}(i)$, para todo $i \in \mathcal{I}$. Agora basta mostrar que o procedimento é bem sucedido.

Para garantir que a simulação perfeita seja bem sucedida, precisamos mostrar que $N_{S T O P}^{(i)}$ é finito quase certamente.

Pelo Teorema 4.3.1, temos que

$$
P\left\{N_{S T O P}^{(i)}>n\right\} \leq \alpha^{n},
$$

onde $\alpha \in(0,1)$.

Isso implica que

$$
\sum_{n=1}^{\infty} P\left\{N_{S T O P}^{(i)}>n\right\}<\infty
$$

Portanto, por Borel Cantelli, temos que

$$
P\left\{N_{S T O P}^{(i)}>n\right\}=0,
$$

para todo $n$ suficientemente grande.

Logo, o Algoritmo 1 é bem sucedido. Portanto, existe um único processo estacionário $\left(\xi_{t}\right)$ assumindo valores em $\mathcal{S}$ com gerador infinitesimal dado por (3.1). Concluindo a prova do Teorema 3.2.1.

\subsection{Prova do Teorema 5.1.1}

Precisamos mostrar que o acoplamento definido no Algoritmo 3 atinge a desigualdade em (5.11). Para isso, precisamos introduzir a seguinte variável. Para $i \in \mathcal{I}$ e $t \geq 0$, defina

$$
T_{1}^{(i, t)}=\inf \left\{T_{n}^{(i)}>t\right\}-t
$$

o primeiro tempo de salto do processo $\left(T_{n}^{(i)}\right)$ logo após o instante $t$, transladado por $t$.

Defina $T_{F}^{(i)}$ o primeiro instante em que um neurônio $j$ que não pertence ao conjunto $F$ é escolhido nos sorteios que definem o conjunto dos ancestrais, dado por

$$
T_{F}^{(i)}=\inf \left\{t>0: C_{t}^{(i)} \cap \bar{F} \neq \emptyset\right\}
$$

onde $\bar{F}$ denota o conjunto complementar de $F$.

Lema 6.3.1. Para qualquer conjunto $F \subset I$ finito $e i \in F$, temos que

$$
T_{F}^{(i)}=\inf \left\{t>0: \sum_{\sigma \in \bar{\Sigma}_{t}(F)} N^{\sigma}\left[t, T_{1}^{(i, t)}[\geq 1\},\right.\right.
$$

onde $\bar{\Sigma}_{t}(F)=\left\{\sigma_{u}^{(j, k)} \in \Sigma: u \in C_{t-}^{(i)}, k \geq 1, j \in V_{\cdot \rightarrow u}^{k} \cap \bar{F}\right\}$.

Demonstração. O evento $\left\{T_{F}^{(i)}=t\right\}$ ocorre se, no instante $t$, para algum $u, k$ e $j$, tais que

$$
u \in C_{t-}^{(i)}, k \geq 1 \text { e } j \in V_{\cdot \rightarrow u}^{k} \cap \bar{F},
$$


temos uma marca do processo $N^{\sigma}$, onde $\sigma=\sigma_{u}^{(j, k)}$. Ou seja, se

$$
\sum_{\sigma \in \bar{\Sigma}_{t}(F)} N^{\sigma}\left[t, t+T^{(i, t)}[\geq 1\right.
$$

Portanto, temos a igualdade em (6.18).

Observe que até o instante $T_{F}^{(i)}$ nenhum potencial de ação de um neurônio no conjunto $\bar{F}$ que possa influenciar a configuração do neurônio $i$ no instante $t=0$ ocorreu. Ou seja, para qualquer $F \subset \mathcal{I}$ e $i \in F$, temos que

$$
C_{s}^{(i),[F]}=C_{s}^{(i)}
$$

para $s<T_{F}^{(i)}$.

Mais ainda, se $T_{S T O P}^{(i)}<T_{F}^{(i)}$ então a saída da simulação perfeita do acoplamento dos processos satisfaz a seguinte igualdade

$$
\xi_{0}^{[F]}(i)=\xi_{0}(i)
$$

Portanto,

$$
P\left\{\xi_{0}(i) \neq \xi_{0}^{[F]}(i)\right\} \leq P\left\{T_{F}^{(i)}<T_{S T O P}^{(i)}\right\}
$$

No próximo lema, encontramos um limitante superior para a probabilidade do evento $\left\{T_{F}^{(i)}<\right.$ $\left.T_{S T O P}^{(i)}\right\}$.

Lema 6.3.2. Para todo $F \subset \mathcal{I}$ finito e $i \in F$, temos que

$$
P\left\{T_{F}^{(i)}<T_{S T O P}^{(i)}\right\} \leq \frac{\delta(F)}{1-\alpha},
$$

onde $\delta(F)$ é definido em (6.26).

Demonstração. Para $F, C \in \mathcal{P}_{f}(\mathcal{I})$, defina

$$
A_{n}(F, C)=\left\{\Pi\left(T_{n-1}^{(i)}, \Sigma_{C}\right)=\sigma, \text { para algum } \sigma \in \bar{\Sigma}_{C}(F)\right\}
$$

onde $\bar{\Sigma}_{C}(F)=\left\{\sigma_{u}^{(j, k)}: u \in C, k \geq 1, j \in V_{\cdot \rightarrow u}^{k} \cap \bar{F}\right\}$ e $\Pi$ é a variável aleatória assumindo valores em $\Sigma$ definida no Capítulo 4.

Note que o evento $\left\{T_{F}^{(i)}<T_{S T O P}^{(i)}\right\}$ é igual a

$$
\bigcup_{n \geq 1} \bigcup_{C \in \mathcal{P}(F)}\left\{A_{n}\left(F, \tilde{C}_{n-1}^{(i)}\right), \tilde{C}_{n-1}^{(i)}=C, N_{S T O P}^{(i)}>n-1\right\} .
$$

Logo, temos que

$$
\begin{aligned}
P\left\{T_{F}^{(i)}<T_{S T O P}^{(i)}\right\} & =P\left\{\bigcup_{n \geq 1} \bigcup_{C \in \mathcal{P}(F)}\left\{A_{n}\left(F, \tilde{C}_{n-1}^{(i)}\right), \tilde{C}_{n-1}^{(i)}=C, N_{S T O P}^{(i)}>n-1\right\}\right\} \\
& \leq \sum_{n \geq 1} \sum_{C \in \mathcal{P}(F)} P\left\{A_{n}\left(F, \tilde{C}_{n-1}^{(i)}\right) \mid \tilde{C}_{n-1}^{(i)}=C, N_{S T O P}^{(i)}>n-1\right\} P\left\{\tilde{C}_{n-1}^{(i)}=C, N_{S T O P}^{(i)}>n-1\right\} \\
& \leq \sum_{n \geq 1} \sum_{C \in \mathcal{P}_{f}(\mathcal{I})} P\left\{A_{n}\left(F, \tilde{C}_{n-1}^{(i)}\right) \mid \tilde{C}_{n-1}^{(i)}=C, N_{S T O P}^{(i)}>n-1\right\} P\left\{\tilde{C}_{n-1}^{(i)}=C, N_{S T O P}^{(i)}>n-1\right\} \\
& =\sum_{n \geq 1} \mathbb{E}\left[P\left\{A_{n}\left(F, \tilde{C}_{n-1}^{(i)}\right) \mid \tilde{C}_{n-1}^{(i)}, N_{S T O P}^{(i)}>n-1\right\}\right] .
\end{aligned}
$$


Dado $\tilde{C}_{n-1}^{(i)}$, temos que

$$
P\left(\Pi\left(T_{n-1}^{(i)}, \tilde{C}_{n-1}^{(i)}\right)=\sigma_{u}^{(j, k)}\right)=\frac{\lambda_{j}(k)}{\sum_{u^{\prime} \in \tilde{C}_{n-1}^{(i)}}},
$$

para $u \in \tilde{C}_{n-1}^{(i)}, k \geq 1$ e $j \in V_{: \rightarrow u}^{k}$.

Somando sobre todas as possibilidades de $u \in \tilde{C}_{n-1}^{(i)}, k \geq 1$ e $j \in V_{\cdot \rightarrow u}^{k}$, temos que

$$
P\left\{A_{n}\left(F, \tilde{C}_{n-1}^{(i)}\right) \mid \tilde{C}_{n-1}^{(i)}, N_{S T O P}^{(i)}>n-1\right\}=q\left(F, \tilde{C}_{n-1}^{(i)}\right) \mathbb{I}\left\{N_{S T O P}^{(i)}>n-1\right\},
$$

onde

$$
q\left(F, \tilde{C}_{n-1}^{(i)}\right)=\frac{\sum_{u \in \tilde{C}_{n-1}^{(i)}} \sum_{k \geq 1} \sum_{j \in V_{\cdot \rightarrow u}^{k}} \lambda_{j}(k) \mathbb{I}\{j \in \bar{F}\}}{\sum_{u^{\prime} \in \tilde{C}_{n-1}^{(i)}} \Lambda_{u^{\prime}}} .
$$

Note que, para todo $C \in \mathcal{P}_{f}(\mathcal{I})$, temos que $q(F, C) \in[0,1]$. Defina, para $F \in \mathcal{P}_{f}(\mathcal{I})$,

$$
\delta(F)=\sup _{C \in \mathcal{P}_{f}(\mathcal{I})} q(F, C) .
$$

Portanto, por (6.25) e (6.26), temos que

$$
\begin{aligned}
P\left\{T_{F}^{(i)}<T_{S T O P}^{(i)}\right\} & \leq \sum_{n \geq 1} \mathbb{E}\left[\delta(F) \mathbb{I}\left\{N_{S T O P}^{(i)}>n-1\right\}\right] \\
& =\delta(F) \sum_{n \geq 1} P\left\{N_{S T O P}^{(i)}>n-1\right\} \\
& =\delta(F) \mathbb{E}\left[N_{S T O P}^{(i)}\right] \\
& =\frac{\delta(F)}{1-\alpha} .
\end{aligned}
$$

Na última igualdade acima, usamos o resultado do Teorema 4.3.1.

Pelo Lema 6.3.2 e pela desigualdade (6.21), temos que

$$
P\left\{\xi_{0}(i) \neq \xi_{0}^{[F]}(i)\right\} \leq \frac{\delta(F)}{1-\alpha},
$$

concluindo a prova do Teorema 5.1.1.

Considere $\left(F_{n}\right)_{n \in \mathbb{N}}$, com $F_{n} \in \mathcal{P}(\mathcal{I})$, uma sequência crescente de subconjuntos de $\mathcal{I}$ tal que

$$
F_{n} \subset F_{n+1} \text {, para } n \geq 0 \text {, e } F_{n} \rightarrow \mathcal{I} \text { quando } n \rightarrow \infty .
$$

Note que para $F=\mathcal{I}, \delta(\mathcal{I})=0$. Como, para $n \geq 0$, temos que $\delta\left(F_{n+1}\right) \leq \delta\left(F_{n}\right)$. Então

$$
\delta\left(F_{n}\right) \rightarrow 0 \text { quando } n \rightarrow \infty .
$$




\section{Capítulo 7}

\section{Conclusão}

No ponto de vista biológico, introduzimos uma nova classe de sistemas markoviano de partículas para a modelagem de sistemas biológicos neurais. Aqui consideramos apenas alguns aspectos da dinâmica de interação entre os neurônios que compõem o sistema.

O modelo não considera o caso dos estímulos inibitórios presentes nesses sistemas. Uma maneira de acrescentar este tipo de estímulo no modelo é considerar uma família de transformações que inclui o caso em que um potencial de ação de um neurônio pode retirar uma quantidade $M \in \mathbb{N}$ de carga positiva do potencial de membrana de um conjunto de neurônios.

A dificuldade em considerarmos esta família de transformações é que não conseguimos distinguir durante a evolução do processo os instantes onde ocorrerem os disparos. O que torna o modelo ambíguo e desinteressante na prática.

No ponto de vista matemático, mostramos a existência e unicidade de um sistema markoviano de partículas em $\mathcal{S}=\mathbb{N}^{\mathcal{I}}$, onde $\mathcal{S}$ é um espaço não compacto. Utilizando a técnica de simulação perfeita, conseguimos mostrar que o processo definido para modelar um sistema biológico neural existe e é único.

Mais ainda, desenvolvemos um pseudo-algoritmo de simulação perfeita para o processo, como também para o acoplamento entre o processo limitado a um conjunto finito de neurônios e o processo que considera todos os neurônios do sistema. 
CONCLUSÃO 


\section{Referências Bibliográficas}

Bertein e Galves(1977) F. Bertein e A. Galves. Une classe de systèmes de particules stable par association. Z. Wahrsheinlichkeitstheorie verw, 41:73-85. Citado na pág. 16, 19

Brillinger(1988) D. Brillinger. Maximum likelihood analysis of spike trains of interacting nerve cells. Biol. Cybern., 59:189-200. Citado na pág. 2

Cessac(2011) B. Cessac. A discrete time neural network model with spiking neurons: II: Dynamics with noise. Journal of Mathematical Biology, 62:863-900. Citado na pág. 1

Comets et al.(2002) F. Comets, R. Fernandez e P. Ferrari. Processes with long memory: regenerative construction and perfect simulation. Annals of Applied Probability, 12:921-943. Citado na pág. 15

Fernández et al.(2001) R. Fernández, A. Galves e P. Ferrari. Coupling, Renewal and Perfect Simulations of Chain of Infnite Order. Lecture Notes for the 5a. Escola Brasileira de Probabilidade, Ubatuba, Brasil. Citado na pág. 7

Fernández et al.(2002) R. Fernández, P. Ferrari e N. Garcia. Perfect simulation for interacting point processes, loss networks and ising models. Stochastic Process. Appl., 102:63-88. Citado na pág. 15

Gallo(2009) S. Gallo. Simulação perfeita de cadeias de alcance variável não limitado. Tese de Doutorado, Instituto de matemática e estatística, Universidade de São Paulo, Brasil. Citado na pág.

Gallo(2011) S. Gallo. Chains with unbounded variable length memory: perfect simulation and visible regeneration scheme. Adv. Appl. Probab., 43:735-759. Citado na pág.

Galves e Löcherbach(2013) A. Galves e E. Löcherbach. Infinite systems of interacting chains with memory of variable length - a stochastic model for biological neural nets. Journal of Statistical Physics, 151(5):896-921. Citado na pág. 1, 2, 3, 6, 8, 9, 16

Galves et al.(2010) A. Galves, E. Löcherbach e E. Orlandi. Perfect Simulation of Infinite Range Gibbs Measures and Coupling with Their Finite Range Approximations. Journal of Statistical Physics, 138:476-495. Citado na pág. 16

Galves et al.(2013) A. Galves, N. Garcia, E. Löcherbach e E. Orlandi. Kalikow-type decomposition for multicolor infinite range particle systems. Annals of Applied Probability, 23:1629-1659. Citado na pág. 16

Gerstner e Kistler(2002) W. Gerstner e W. M. Kistler. Spiking Neuron Models. Sinlge Neurons, Populations, Plasticity. Cambridge University Press. Citado na pág. 3, 8

Goldberg et al.(1955) J.M. Goldberg, H.O. Adrian e F.D. Smith. Response of neurons of the superior olivary complex of the cat to acoustic stimuli of long duration. J. Neurophysiol., 27: 707-724. Citado na pág. 3 
Hawkes(1971) A. Hawkes. Point spectra of some mutually exciting point processes. J. R. Stat. Soc., 33:438-443. Citado na pág. 2

Liggett(1999) T. Liggett. Stochastic Interacting Systems: Contact, Voter and Exclusion Processe. Springer. Citado na pág.

Nawrot et al.(2007) M.P. Nawrot, C. Boucsein, V. Rodriguez-Molina, A. Aertsen, S. Grün e S. Rotter. Serial interval statistics of spontaneous activity in cortical neurons in vivo and in vitro. Neurocomputing, 70:1717-1722. Citado na pág. 3, 8

Propp e Wilson(1995) J. G. Propp e D. B. Wilson. Exact sampling with coupled Markov chains and applications to statistical mechanics. Em Proceedings of the Seventh International Conference on Random Structures and Algorithms (Atlanta, GA, 1995), volume 9. Citado na pág. 15

Spitzer(1970) F. Spitzer. Interaction of Markov processes. Adv. Math., 5:290-290. Citado na pág. 